\title{
Non-relativistic and Carrollian limits of Jackiw-Teitelboim gravity
}

\author{
Joaquim Gomis, ${ }^{a}$ Diego Hidalgo ${ }^{b, c, d}$ and Patricio Salgado-Rebolledo ${ }^{e}$ \\ ${ }^{a}$ Departament de Física Quàntica i Astrofísica and Institut de Ciències del Cosmos (ICCUB), \\ Universitat de Barcelona, \\ Martí i Franquès 1, E-08028 Barcelona, Spain \\ ${ }^{b}$ Centro de Estudios Científicos (CECs), \\ Av. Arturo Prat 514, Valdivia, Chile \\ ${ }^{c}$ Departamento de Física, Universidad de Concepción, \\ Casilla 160-C, Concepción, Chile \\ ${ }^{d}$ Instituto de Ciencias Físicas y Matemáticas, Universidad Austral de Chile, \\ Casilla 567, Valdivia, Chile \\ ${ }^{e}$ Université Libre de Bruxelles and International Solvay Institutes, \\ ULB-Campus Plaine CP231, B-1050 Brussels, Belgium \\ E-mail: joaquim.gomis@ub.edu, dhidalgo@cecs.cl, psalgado@ulb.ac.be
}

ABSTRACT: We construct the non-relativistic and Carrollian versions of Jackiw-Teitelboim gravity. In the second order formulation, there are no divergences in the non-relativistic and Carrollian limits. Instead, in the first order formalism, some divergences can be avoided by starting from a relativistic BF theory with $(\mathrm{A}) \mathrm{dS}_{2} \times \mathbb{R}$ gauge algebra. We show how to define the boundary duals of the gravity actions using the method of non-linear realisations and suitable Inverse Higgs constraints. In particular, the non-relativistic version of the Schwarzian action is constructed in this way. We derive the asymptotic symmetries of the theory, as well as the corresponding conserved charges and Newton-Cartan geometric structure. Finally, we show how the same construction applies to the Carrollian case.

KeYwords: 2D Gravity, Classical Theories of Gravity, Space-Time Symmetries, Topological Field Theories

ARXIV EPRINT: 2011.15053 


\section{Contents}

1 Introduction 1

2 Jackiw-Teitelboim gravity 3

2.1 Jackiw-Teitelboim action 4

2.2 First order formulation of JT gravity 4

3 Non-relativistic Jackiw-Teitelboim gravity $\quad 6$

3.1 Second order formulation $\quad 6$

$\begin{array}{lll}3.2 & \text { First order formulation } & 10\end{array}$

4 Carrollian Jackiw-Teitelboim gravity $\quad 13$

$\begin{array}{lll}4.1 \text { Second order formulation } & 13\end{array}$

$\begin{array}{lll}4.2 & \text { First order formulation } & 14\end{array}$

5 Relativistic boundary theory $\quad \mathbf{1 6}$

$\begin{array}{lll}5.1 & (\mathrm{~A}) \mathrm{dS}_{2} \text { case } & 18\end{array}$

5.2 Derivation of the boundary action in second order formalism 22

$5.3(\mathrm{~A}) \mathrm{dS}_{2} \times \mathbb{R}$ case $\quad 23$

6 Non-relativistic boundary theory $\quad 27$

6.1 Non-relativistic limit of the $\operatorname{SL}(2, \mathbb{R}) \times \mathbb{R}$ boundary action $\quad 27$

$\begin{array}{lll}6.2 & \text { Extended Newton-Hooke } \\ 2 & \text { case } & 29\end{array}$

$\begin{array}{lll}6.3 & \text { Extended Newton-Hooke } \\ 2 & \text { case } & 32\end{array}$

6.4 Extended Carroll (A) $\mathrm{dS}_{2}$ case 33

7 Conclusions and outlook $\quad 34$

$\begin{array}{ll}\text { A Conventions for }(\mathrm{A}) \mathrm{dS}_{2} & 35\end{array}$

B Building blocks for the NR second order formulation 36

$\begin{array}{lll}\text { B.1 NR affine connections } & 36\end{array}$

$\begin{array}{lll}\text { B.2 Ricci scalar } & 37\end{array}$

\section{Introduction}

The Sachdev-Ye-Kitaev (SYK) model [1-4] is a solvable quantum mechanical model of Majorana fermions in one dimension. In the regime of large coupling (low temperature) this model is perturbative in the $1 / N$-expansion and shows emergent conformal symmetry, which is the reparametrisation symmetry group $\operatorname{Diff}\left(S^{1}\right)$. The one-dimensional diffeomorphism symmetry is spontaneously broken to $\mathrm{SL}(2, R)$, whose low energy dynamics are described by the 1D Schwarzian theory $[4,7,8]$. The low energy dynamics of the SYK model can be holographically described by the Jackiw-Teitelboim (JT) gravity [5, 6] in 
two dimensions since the boundary description of the bulk JT gravity is also given by the Schwarzian action [9-11]. The SYK model has been generalised to include complex fermions and its low energy dynamics [12-17] is described by a generalised Schwarzian theory with symmetry $\mathrm{SL}(2, R) \times \mathrm{U}(1)$. A flat limit of this generalised Schwarzian action was studied in [18]. JT gravity and its boundary description are also useful for the momentum/complexity correspondence and its connection with the non-relativistic Newton's law $[19-21]$.

Motivated by the previous ideas and with the goal of finding another physical sector of the SYK model and its holographic description, ${ }^{1}$ we will consider the non-relativistic (NR) and Carrollian limit of JT gravity and its boundary action. The starting point of this study consists of writing the JT gravity action as a BF theory with (A) $\mathrm{dS}_{2}$ gauge group [24-26]. Then, as shown in [27], the Schwarzian action can also be obtained via the method of non-linear realisations [28, 29] and the inverse Higgs mechanism (IHM) [30]. However, in order to have a finite NR limit we will need to extend the analysis and consider a BF theory with gauge group $(\mathrm{A}) \mathrm{dS}_{2} \times \mathbb{R}$. The NR and Carrollian bulk actions can also be expressed as BF theories with Newton-Hooke ${ }^{2}\left(\mathrm{NH}_{2}^{ \pm}\right)$and Carroll $(\mathrm{A}) \mathrm{dS}_{2}$ gauge algebras, respectively [31-33].

To study the NR boundary theory we should consider the conformal basis of $(\mathrm{A}) \mathrm{dS}_{2} \times \mathbb{R}$, and introduce two different contractions of the $\mathrm{SL}(2, \mathbb{R}) \times \mathbb{R}$ algebra. The first one leads to

$$
[\mathcal{H}, \mathcal{D}]=\mathcal{H}, \quad[\mathcal{K}, \mathcal{D}]=-\mathcal{K}, \quad[\mathcal{H}, \mathcal{K}]=2 \mathcal{Z},
$$

which we name the Extended Galilean conformal algebra in 1D dimension. This algebra is isomorphic to $\mathrm{NH}^{+}$. The second contraction gives

$$
[\hat{\mathcal{H}}, \hat{\mathcal{D}}]=\hat{\mathcal{K}}, \quad[\hat{\mathcal{K}}, \hat{\mathcal{D}}]=-\hat{\mathcal{H}}, \quad[\hat{\mathcal{H}}, \hat{\mathcal{K}}]=2 \hat{\mathcal{Z}},
$$

which is isomorphic to $\mathrm{NH}^{-}$and we will refer to it as Twisted Extended Galilean conformal algebra in $1 D$ dimension. These two algebras are isomorphic as complex algebras.

The boundary actions are constructed via the non-linear realisation method and IHM. In the case of a NR limit of the $\mathrm{dS}_{2} \times \mathbb{R}$, the boundary action has Extended Galilean conformal symmetry. We name this action Non-Relativistic Schwarzian. Instead, if we consider the NR limit of the $\mathrm{AdS}_{2} \times \mathbb{R}$ algebra, the boundary action has Twisted Extended Galilean conformal symmetry. It is a complex version of the NR Schwarzian which is closely related to the flat Schwarzian action of [18].

The Carrollian limit of the relativistic bulk and boundary actions is obtained from the observation that the Carroll $(\mathrm{A}) \mathrm{dS}_{2}$ algebra admits a central extension in the commutator of the Galilean boost and momentum generator, ${ }^{3}$ we name this algebra Extended Carroll

\footnotetext{
${ }^{1}$ In the case of the original AdS/CFT duality, two interesting sectors have been considered: the BMN [22] and the one associated to the string non-relativistic limit of $A d S_{5} \times S^{5}$ [23].

${ }^{2}$ The plus sign corresponds to the contraction of dS algebra while the minus sign corresponds to the contraction of AdS algebra. Note that, the space-time symmetries of the harmonic oscillator form the $\mathrm{NH}_{2}^{+}$ algebra, whereas the inverted harmonic oscillator has a $\mathrm{NH}_{2}^{-}$symmetry algebra.

${ }^{3}$ The existence of this extension is a unique feature of the two-dimensional case since, unlike the Galilean case, the Carroll algebra does not admit a non-trivial central extension in four dimensions.
} 
(A) $d S_{2}$ algebra. One can see that this symmetry follows from the Extended $\mathrm{NH}_{2}^{ \pm}$algebra by interchanging the generators $H$ and $P$ and changing the sign of the cosmological constant. This fact allows one to pass from Galilean to Carrollian symmetries ${ }^{4}$

$$
\begin{aligned}
& \text { Extended } \mathrm{NH}_{2}^{+} \leftrightarrow \text { Extended Carroll } \mathrm{AdS}_{2} \\
& \text { Extended } \mathrm{NH}_{2}^{-} \leftrightarrow \text { Extended Carroll } \mathrm{dS}_{2} .
\end{aligned}
$$

The Carrollian actions in the bulk and the boundary can therefore be obtained from the NR ones.

The IHM allows us to construct the boundary BF gauge fields. Similarly as done in 3D gravity [34], it is possible to further reconstruct bulk gauge fields, from which we can construct Newton-Cartan [35-40] and Carrollian geometric structures [41, 42].

The NR and Carrollian limits of the JT bulk action $[5,6]$ in the second order formalism are also studied. In this case, the divergences in the NR and Carrollian limits can be absorbed by rescaling the Newton constant. This is because in two dimensions there are no divergent terms in the expansion of the Ricci scalar, which is a remarkable property that is in high contrast with its analog in three and four space-time dimensions [43-46].

The organisation of the paper is as follows: in section 2, we review the main aspects of JT gravity in first and second order formulations. In section 3, we study the NR limit of the JT action, first in the second order formulation, and subsequently in the first order formulation by considering a BF theory with $(\mathrm{A}) \mathrm{dS}_{2} \times \mathbb{R}$ gauge algebra. section 4 is devoted to the Carrollian limit of the JT gravity theory, which is carried out by using the duality among NR and Carrollian symmetries. Section 5 deals with the construction of the boundary theory of JT gravity. Here we provide a derivation of the known Schwarzian theory and its asymptotic symmetries using the non-linear realisation method and IHM. We generalize the procedure to the $(A) d S_{2} \times \mathbb{R}$ case. In section 6 , we define the $\mathrm{NR}$ Schwarzian action and its asymptotic symmetries and conserved charges. We show how this is generalized to the Carrollian case. Finally, in section 7, we give our conclusions and elaborate on relevant future directions and possible generalizations of our results.

Note added. When this paper was finished, we noticed in the arXiv the article [47], "Limits of JT gravity" by D. Grumiller, J. Hartong, S. Prohazka and J. Salzer. Some of the results of this paper coincide with ours. However, the techniques used in the derivations are different.

\section{Jackiw-Teitelboim gravity}

In this section, we review certain aspects of the Jackiw-Teitelboim (JT) gravity $[5,6]$ which is dual to the SYK model [1-4] in $(0+1)$-dimensions. We start considering the second order formulation of JT gravity and subsequently review its first order formulation.

\footnotetext{
${ }^{4}$ This relation generalises the known duality between Galilean and Carrollian symmetries [48] in the flat case, see also $[42,49]$.
} 


\section{$2.1 \quad$ Jackiw-Teitelboim action}

The JT action for gravity in $(1+1)$-dimensions is given by

$$
S_{\mathrm{JT}}=\tilde{\kappa} \int d^{2} x \sqrt{-g} \Phi(\mathcal{R}-2 \tilde{\Lambda}),
$$

where $\mathcal{R}$ is the Ricci scalar, $\tilde{\Lambda}$ is the cosmological constant, $\Phi$ a scalar field, and $\tilde{\kappa}$ a twodimensional coupling constant. The field equations that follow from varying $\Phi$ and $g_{\mu \nu}$ in (2.1) read

$$
\begin{aligned}
\mathcal{R}-2 \tilde{\Lambda} & =0 \\
\left(\nabla_{\mu} \nabla_{\nu}-g_{\mu \nu} \nabla^{2}\right) \Phi+\tilde{\Lambda} g_{\mu \nu} \Phi & =0
\end{aligned}
$$

where $\nabla_{\mu}$ denotes the affine space-time covariant derivative. The second equation can be decomposed into traceless and trace parts as [50]

$$
\begin{aligned}
\left(\nabla_{\mu} \nabla_{\nu}-\frac{1}{2} g_{\mu \nu} \nabla^{2}\right) \Phi & =0, \\
\left(\nabla^{2}-2 \tilde{\Lambda}\right) \Phi & =0,
\end{aligned}
$$

where we recognize (2.3b) as the Klein-Gordon equation on $(\mathrm{A}) \mathrm{dS}_{2}$. Using this equation, one can see that $(2.2 \mathrm{~b})$ is equivalent to

$$
\nabla_{\mu} \nabla_{\nu} \Phi-\tilde{\Lambda} g_{\mu \nu} \Phi=0
$$

In the following, we will review how the above geometric dynamics may be presented in a gauge theoretical fashion.

\subsection{First order formulation of JT gravity}

A first order formulation of JT gravity can be defined by gauging the (A) $\mathrm{dS}_{2}$ symmetry and constructing a BF theory [24-26]. The gauge algebra is given by $\mathfrak{s o}(1,2)$ in the AdS case and $\mathfrak{s o}(2,1)$ for $\mathrm{dS}$. The generators satisfy the following commutation relations ${ }^{5}$

$$
\left[\tilde{J}, \tilde{P}_{a}\right]=\epsilon_{a}{ }^{b} \tilde{P}_{b}, \quad\left[\tilde{P}_{a}, \tilde{P}_{b}\right]=-\tilde{\Lambda} \epsilon_{a b} \tilde{J}
$$

where $\tilde{P}_{a}$ stand for translations and $\tilde{J}$ is the boost generator. The invariant tensor in (A) $\mathrm{dS}_{2}$ is given by

$$
\langle\tilde{J}, \tilde{J}\rangle=\tilde{\gamma}_{0}, \quad\left\langle\tilde{P}_{a}, \tilde{P}_{b}\right\rangle=-\tilde{\gamma}_{0} \tilde{\Lambda} \eta_{a b},
$$

where $\tilde{\gamma}_{0}$ is an arbitrary constant.

In order to define a BF theory we consider a scalar field $B$ taking values in the (A) $\mathrm{dS}_{2}$ algebra

$$
B=\Phi^{a} \tilde{P}_{a}+\Phi \tilde{J}
$$

\footnotetext{
${ }^{5}$ For the conventions see appendix A.
} 
and a set of one-form gauge fields, $E^{a}=E_{\mu}^{a} d x^{\mu}$ and $\Omega=\Omega_{\mu} d x^{\mu}$, corresponding to the zweibein form and the dual spin connection $\Omega \equiv-\frac{1}{2} \varepsilon_{a b} \Omega^{a b}$, respectively. The gauge fields define the $(\mathrm{A}) \mathrm{dS}_{2}$ connection one form

$$
A=E^{a} \tilde{P}_{a}+\Omega \tilde{J}
$$

together with the corresponding curvature two-form $F=d A+A^{2}$, given by ${ }^{6}$

$F=R^{a}(\tilde{P}) \tilde{P}_{a}+R(\tilde{J}) \tilde{J}, \quad R^{a}(\tilde{P})=d E^{a}-\epsilon_{b}^{a} \Omega E^{b}, \quad R(\tilde{J})=d \Omega-\frac{\tilde{\Lambda}}{2} \epsilon_{a b} E^{a} E^{b}$.

The BF action then reads

$$
S[B, A]=\int\langle B, F\rangle=\tilde{\gamma}_{0} \int\left(\Phi R(\tilde{J})-\tilde{\Lambda} \Phi_{a} R^{a}(\tilde{P})\right),
$$

and its field equations are given by

$$
\begin{array}{rlrl}
\delta \Phi^{a}: & & R^{a}(\tilde{P}) & =0, \\
\delta \Phi: & R(\tilde{J}) & =0, \\
\delta E^{a}: & d \Phi^{a}-\epsilon_{b}^{a}\left(\Phi E^{b}+\Omega \Phi^{b}\right) & =0, \\
\delta \Omega: & d \Phi-\tilde{\Lambda} \epsilon_{a b} E^{a} \Phi^{b} & =0 .
\end{array}
$$

From equation (2.11a) we can solve the spin connection in terms of the zweibein as

$$
\Omega_{\mu}(E)=-E^{-1} \epsilon^{\alpha \beta} \partial_{\alpha} E_{\beta}^{a} E_{a \mu},
$$

where

$$
E \equiv \operatorname{det}\left(E_{\mu}^{a}\right)=-\frac{1}{2} \epsilon^{\mu \nu} \epsilon_{a b} E_{\mu}^{a} E_{\nu}^{b}
$$

Using this result, one can evaluate the Riemann tensor

$$
\mathcal{R}(E)^{a}{ }_{b \rho \sigma}=E_{\mu}^{a} E_{b}^{\nu} \mathcal{R}(E)^{\mu}{ }_{\nu \rho \sigma}=-\epsilon_{b}^{a}\left(\partial_{\rho} \Omega(E)_{\sigma}-\partial_{\sigma} \Omega(E)_{\rho}\right),
$$

which indicates that the field equation $(2.11 \mathrm{~b})$ can be rewritten in terms of the Ricci scalar $\mathcal{R}=g^{\mu \nu} \mathcal{R}_{\mu \rho \nu}^{\rho}$ as

$$
\epsilon^{\mu \nu}\left(\partial_{\mu} \Omega(E)_{\nu}-\frac{\tilde{\Lambda}}{2} \epsilon_{a b} E_{\mu}^{a} E_{\nu}^{b}\right)=-\frac{E}{2}(\mathcal{R}(E)-2 \tilde{\Lambda})=0,
$$

and reproduces the field equation (2.2a) found in the second order formulation. On the other hand, from (2.11d) we can express $\Phi^{a}$ in terms of $\Phi$ as

$$
E_{a}^{\mu} \Phi^{a}=\frac{E^{-1}}{\tilde{\Lambda}} \epsilon^{\mu \nu} \partial_{\nu} \Phi
$$

\footnotetext{
${ }^{6}$ Wedge product between differential forms is assumed.
} 
Using this equation in $(2.11 \mathrm{~d})$ yields

$$
\nabla_{\mu} \nabla_{\nu} \Phi-\tilde{\Lambda} g_{\mu \nu} \Phi=0
$$

where the space-time metric is defined in the usual way

$$
g_{\mu \nu}=E_{\mu}^{a} E_{\nu}^{b} \eta_{a b} .
$$

This reproduces the field equation (2.4) previously found in the second order formulation. Using (2.11a) and (2.15), the BF action (2.10) takes the form of the JT action (2.1), namely

$$
S[B, A]=-\frac{\tilde{\gamma}_{0}}{2} \int d^{2} x E \Phi(\mathcal{R}(E)-2 \tilde{\Lambda}),
$$

where we have to identify $\tilde{\gamma}_{0}=-2 \tilde{\kappa}$.

However, since the spin connection was not solved by means of its own field equation, the reduced action (2.19) is not dynamically equivalent to the original BF action (2.10). Thus, the equivalence between the first order and second order formulations of JT gravity should be considered only at the level of their field equations. The solutions in the secondorder formalism are contained as a subset of the solutions of the first-order formalism.

\section{Non-relativistic Jackiw-Teitelboim gravity}

In this section, we will consider the NR limit of JT gravity in second and first order formulations. In the first order formulation, the limit is applied to a BF theory with gauge group $(\mathrm{A}) \mathrm{dS}_{2} \times \mathbb{R}$.

\subsection{Second order formulation}

The NR expansion of JT gravity follows in general terms from the approaches studied in $[39,43-45,51-54]$. Let us consider the power expansion of the relativistic metric $g_{\mu \nu}(x)$ and $g^{\mu \nu}(x)$ up to $\varepsilon^{2}$-order, as follows

$$
g_{\mu \nu}=\frac{1}{\varepsilon^{2}} \stackrel{(2)}{g}_{\mu \nu}+\stackrel{(0)}{g}_{\mu \nu}+\mathcal{O}\left(\varepsilon^{2}\right), \quad g^{\mu \nu}=\stackrel{(0)}{g}^{\mu} \mu \nu+\varepsilon^{2} \stackrel{(-2)}{g}_{\mu \nu}+\mathcal{O}\left(\varepsilon^{4}\right),
$$

where the higher inverse powers of $\varepsilon=1 / c \rightarrow 0$ correspond to Post-Newtonian corrections that will not be considered here. From the relation $g_{\mu \rho} g^{\rho \nu}=\delta_{\mu}^{\nu}$, we obtain the following relations for the different terms in the expansion

$$
\stackrel{(2)}{g}_{\mu \nu} \stackrel{(-2)}{g} \nu \rho+\stackrel{(0)}{g}_{\mu \nu} \stackrel{(0)}{g} \nu \rho=\delta_{\mu}^{\rho}, \quad \stackrel{(2)}{g}_{\mu \nu} \stackrel{(0)}{g} \nu \rho=0, \quad \stackrel{(0)}{g}_{\mu \nu} \stackrel{(-2)}{g} \nu \rho=0
$$

Now we introduce a symmetric affine Levi-Civita connection with the expansion ${ }^{7}$

$$
\Gamma_{\mu \nu}^{\lambda}=\frac{1}{\varepsilon^{2}} \stackrel{(2)}{\Gamma}{ }_{\mu \nu}^{\lambda}+\stackrel{(0)}{\Gamma}{ }_{\mu \nu}^{\lambda}+\varepsilon^{2} \stackrel{(-2)}{\Gamma} \underset{\mu \nu}{\lambda} .
$$

\footnotetext{
${ }^{7}$ Notice that here we could consider a more general affine connection, including an antisymmetric part related to the torsion in Lorentzian geometry, but we do not consider that case.
} 
As usual, we demand metric compatibility on the metric and its inverse

$$
\nabla_{\rho} g_{\mu \nu}=0, \quad \nabla_{\rho} g^{\mu \nu}=0,
$$

where the covariant derivative $\nabla_{\rho}$ is defined with respect to the symmetric connection (3.3). For the covariant metric $g_{\mu \nu}$, this condition implies the following set of equations order by order

$$
\begin{aligned}
& \stackrel{(0)}{\nabla}_{\rho} \stackrel{(0)}{g}_{\mu \nu}-\stackrel{(-2)}{\Gamma}_{\rho \nu}^{\lambda} \stackrel{(2)}{g}_{\mu \lambda}-\stackrel{(-2)}{\Gamma}_{\rho \mu}^{\lambda} \stackrel{(2)}{g}{ }_{\nu \lambda}=0, \\
& \stackrel{(0)}{\nabla}_{\rho} \stackrel{(2)}{g}_{\mu \nu}-\stackrel{(2)}{\Gamma}_{\rho \nu}^{\lambda} \stackrel{(0)}{g}_{\mu \lambda}-\stackrel{(2)}{\Gamma}_{\rho \mu}^{\lambda} \stackrel{(0)}{g}_{\nu \lambda}=0, \\
& \stackrel{(2)}{\Gamma}_{\rho \nu}^{\lambda} \stackrel{(2)}{g}_{\mu \lambda}+\stackrel{(2)}{\Gamma}_{\rho \mu}^{\lambda} \stackrel{(2)}{g}_{\nu \lambda}=0, \\
& \stackrel{(-2)}{\Gamma}_{\rho \nu} \stackrel{(0)}{g}_{\mu \lambda}+\stackrel{(-2)}{\Gamma}_{\rho \mu}^{\lambda} \stackrel{(0)}{g}_{\nu \lambda}=0 \text {, }
\end{aligned}
$$

where we have introduced the covariant derivative $\stackrel{(0)}{\nabla} \equiv \partial+\stackrel{(0)}{\Gamma}$. Solving the system yields (see appendix B.1)

$$
\begin{aligned}
\stackrel{(2)}{\Gamma}_{\mu \nu}^{\lambda}= & \frac{1}{2} \stackrel{(0)}{g} \lambda \sigma\left(\partial_{\nu} \stackrel{(2)}{g}_{\sigma \mu}+\partial_{\mu} \stackrel{(2)}{g}_{\sigma \nu}-\partial_{\sigma} \stackrel{(2)}{g}_{\mu \nu}\right), \\
\stackrel{(0)}{\Gamma}_{\mu \nu}^{\lambda}= & \frac{1}{2} \stackrel{(-2)}{g} \lambda \sigma\left(\partial_{\nu} \stackrel{(2)}{g}_{\sigma \mu}+\partial_{\mu} \stackrel{(2)}{g}_{\sigma \nu}-\partial_{\sigma} \stackrel{(2)}{g}_{\mu \nu}\right) \\
& +\frac{1}{2} \stackrel{(0)}{g} \lambda \sigma\left(\partial_{\nu} \stackrel{(0)}{g}_{\sigma \mu}+\partial_{\mu} \stackrel{(0)}{g}_{\sigma \nu}-\partial_{\sigma} \stackrel{(0)}{g}_{\mu \nu}\right), \\
\stackrel{(-2)}{\Gamma}_{\mu \nu}^{\lambda}= & \frac{1}{2} \stackrel{(-2)}{g} \lambda \sigma\left(\partial_{\nu} \stackrel{(0)}{g}_{\sigma \mu}+\partial_{\mu} \stackrel{(0)}{g}_{\sigma \nu}-\partial_{\sigma} \stackrel{(0)}{g}_{\mu \nu}\right) .
\end{aligned}
$$

Now we write the metric (2.18) in terms of Newton-Cartan fields $\tau_{\mu}$ and $e_{\mu}$, and the vector field $m_{\mu}$ by considering the zweibein expansion [35-40]

$$
E_{\mu}^{0}=\frac{1}{\varepsilon} \tau_{\mu}+\frac{\varepsilon}{2} m_{\mu}, \quad E_{\mu}^{1}=e_{\mu},
$$

where $m_{\mu}$ is the vector field associated to the central extension of the Bargmann algebra [44, 56]. This leads to

$$
\stackrel{(2)}{g}_{\mu \nu}=-\tau_{\mu} \tau_{\nu}, \quad \stackrel{(0)}{g}_{\mu \nu}=h_{\mu \nu}-\tau_{(\mu} m_{\nu)},
$$

where we have introduced the spatial metric $h_{\mu \nu} \equiv e_{\mu} e_{\nu}$. The relations $E_{a}^{\mu} E_{\nu}^{a}=\delta_{\nu}^{\mu}$, and $E_{b}^{\mu} E_{\mu}^{a}=\delta_{b}^{a}$ lead to the following inverse zweibeine

$$
E_{0}^{\mu}=\varepsilon \tau^{\mu}-\frac{\varepsilon^{3}}{2} \tau^{\mu} \tau^{\nu} m_{\nu}, \quad E_{1}^{\mu}=e^{\mu}-\frac{\varepsilon^{2}}{2} \tau^{\mu} e^{\nu} m_{\nu},
$$

which involve the new additional fields $\tau^{\mu}$ and $e^{\mu}$ satisfying the relations

$$
e^{\mu} e_{\nu}+\tau^{\mu} \tau_{\nu}=\delta_{\nu}^{\mu}, \quad \tau^{\mu} e_{\mu}=0, \quad e^{\mu} \tau_{\mu}=0, \quad \tau_{\mu} \tau^{\mu}=1, \quad e^{\mu} e_{\mu}=1
$$


Note that the spatial metric $h_{\mu \nu}$ is degenerate. With these definitions the inverse metric takes the form

$$
\begin{aligned}
g^{\mu \nu} & =\eta^{a b} E_{a}^{\mu} E_{b}^{\nu} \\
& =e^{\mu} e^{\nu}-\varepsilon^{2} \tau^{\mu} \tau^{\nu}-\frac{\varepsilon^{2}}{2}\left(\tau^{\mu} e^{\nu}+e^{\mu} \tau^{\nu}\right) e^{\sigma} m_{\sigma}+\varepsilon^{4} \tau^{\mu} \tau^{\nu} e^{\sigma} e^{\rho} m_{\sigma} m_{\rho}
\end{aligned}
$$

Therefore, considering terms only up to order $\varepsilon^{2}$, we obtain the following form of the inverse temporal and spatial metrics in (3.1),

$$
\stackrel{(0)}{g} \mu \nu=e^{\mu} e^{\nu} \equiv h^{\mu \nu}, \quad \stackrel{(-2)}{g} \mu \nu=-\tau^{\mu} \tau^{\nu}-\tau^{(\nu} h^{\mu) \sigma} m_{\sigma},
$$

where we have introduced the inverse spatial metric $h^{\mu \nu}$. With these explicit forms for the metric components, the terms in the connection expansion (3.6) take the form

$$
\begin{aligned}
& \stackrel{(2)}{\Gamma}{ }_{\mu \nu}^{\lambda}=h^{\lambda \sigma}\left(\tau_{\mu} \partial_{[\sigma} \tau_{\nu]}+\tau_{\nu} \partial_{[\sigma} \tau_{\mu]}\right) \\
& \stackrel{(0)}{\Gamma}{ }_{\mu \nu}^{\lambda}=\tau^{\lambda} \partial_{(\mu} \tau_{\nu)}+\frac{1}{2} h^{\lambda \sigma}\left(\partial_{\nu} h_{\sigma \mu}+\partial_{\mu} h_{\sigma \nu}-\partial_{\sigma} h_{\mu \nu}\right)+h^{\lambda \sigma}\left(\partial_{[\sigma} m_{\mu]} \tau_{\nu}+\partial_{[\sigma} m_{\nu]} \tau_{\mu}\right) \\
& \stackrel{(-2)}{\Gamma} \underset{\mu \nu}{\lambda}=-\frac{1}{2}\left(\tau^{\lambda} \tau^{\sigma}+\tau^{(\lambda} h^{\sigma) \rho} m_{\rho}\right)\left(\partial_{\nu}\left(h_{\sigma_{\mu}}-\tau_{(\sigma} m_{\mu)}\right)\right. \\
&\left.+\partial_{\mu}\left(h_{\sigma_{\nu}}-\tau_{(\sigma} m_{\nu)}\right)-\partial_{\sigma}\left(h_{\mu_{\nu}}-\tau_{(\mu} m_{\nu)}\right)\right)
\end{aligned}
$$

Given (3.1), the corresponding expansion of the Ricci scalar $\mathcal{R}$ is (see appendix B.2)

$$
\mathcal{R}=\varepsilon^{2} \stackrel{(-2)}{\mathcal{R}}+\mathcal{O}\left(\varepsilon^{4}\right)
$$

We see that in two dimensions there are no divergent terms in the expansion of the Ricci scalar. This is a remarkable property that is in high contrast with three and four space-time dimensions [43-46].

We consider now the expansion of the vielbein postulate,

$$
\partial_{\mu} E_{\lambda}^{a}+\varepsilon_{b}^{a} \Omega_{\mu} E_{\lambda}^{b}-\Gamma_{\mu \lambda}^{\rho} E_{\rho}^{a}=0 .
$$

Implementing (3.3), (3.9) and considering

$$
\Omega_{\mu}=\varepsilon \omega_{\mu},
$$

the anti-symmetric part of $(3.15)$ is

$$
\begin{aligned}
\partial_{[\mu} \tau_{\lambda]} & =0, \\
\partial_{[\mu} e_{\lambda]}+\omega_{[\mu} \tau_{\lambda]} & =0,
\end{aligned}
$$

$$
\begin{aligned}
\partial_{[\mu} m_{\lambda]}+\omega_{[\mu} e_{\lambda]} & =0, \\
\omega_{[\mu} m_{\lambda]} & =0,
\end{aligned}
$$


whereas the symmetric part reads

$$
\begin{aligned}
\stackrel{(2)}{\Gamma}_{\mu \lambda}^{\rho} \tau_{\rho} & =0, \\
\stackrel{(2)}{\Gamma}_{\mu \lambda}^{\rho} e_{\rho} & =0, \\
\partial_{\mu} \tau_{\lambda}-\stackrel{(2)}{\Gamma}_{\mu \lambda}^{\rho} m_{\rho}-\stackrel{(0)}{\Gamma}_{\mu \lambda}^{\rho} \tau_{\rho} & =0, \\
\partial_{\mu} m_{\lambda}+\omega_{\mu} e_{\lambda}-\stackrel{(0)}{\Gamma}_{\mu \lambda}^{\rho} m_{\rho}-\stackrel{(-2)}{\Gamma}_{\mu \lambda} \tau_{\rho} & =0, \\
\partial_{\mu} e_{\lambda}+\omega_{\mu} \tau_{\lambda}-\stackrel{(0)}{\Gamma}_{\mu \lambda}^{\rho} e_{\rho} & =0, \\
\omega_{\mu} m_{\lambda}-\stackrel{(-2)}{\Gamma}_{\rho}{ }_{\mu \lambda} & e_{\rho}=0 .
\end{aligned}
$$

Solving this system for all the connections, yields

$$
\begin{aligned}
& \stackrel{(2)}{\Gamma}_{\mu \lambda}^{\rho}=0 \\
& \stackrel{(0)}{\Gamma}_{\mu \lambda}^{\rho}=\tau^{\rho} \partial_{\mu} \tau_{\lambda}+e^{\rho}\left(\partial_{\mu} e_{\lambda}+\omega_{\mu} \tau_{\lambda}\right) \\
& \stackrel{(-2)}{\Gamma} \underset{\mu \lambda}{\rho}=\tau^{\rho}\left[\partial_{\mu} m_{\lambda}+\omega_{\mu} e_{\lambda}-\left(\tau^{\sigma} \partial_{\mu} \tau_{\lambda}+e^{\sigma}\left(\partial_{\mu} e_{\lambda}+\omega_{\mu} \tau_{\lambda}\right)\right) m_{\sigma}\right]+e^{\rho} \omega_{\mu} m_{\lambda} .
\end{aligned}
$$

which is compatible with (3.13). Using this result, the leading term in the Ricci scalar expansion (3.14) takes the form (see appendix B for details),

$$
\stackrel{(-2)}{\mathcal{R}}=4 \tau^{\mu} e^{\nu} \partial_{[\mu} \omega_{\nu]},
$$

where the NR spin connection $\omega_{\mu}$ is solved algebraically by equations (3.17c) and (3.17d), yielding

$$
\omega_{\mu}=2 \tau^{[\alpha} e^{\beta]}\left(e_{\mu} \partial_{\alpha} e_{\beta}-\tau_{\mu} \partial_{\alpha} m_{\beta}\right) .
$$

Finally, we expand the metric determinant, the JT scalar field, the cosmological constant, and the coupling constant as

$$
\begin{aligned}
& \sqrt{-g}=\operatorname{det}\left(E_{\mu}^{a}\right)=-\epsilon^{\mu \nu} E_{\mu}{ }^{0} E_{\nu}{ }^{1}=\frac{1}{\varepsilon} \operatorname{det}(\tau e)+\mathcal{O}(\varepsilon), \\
& \Phi=\varepsilon \phi, \quad \tilde{\Lambda}=\varepsilon^{2} \Lambda \quad \tilde{\kappa}=\frac{1}{\varepsilon^{2}} \kappa .
\end{aligned}
$$

where we have defined

$$
\operatorname{det}(\tau e) \equiv-\epsilon^{\mu \nu} \tau_{\mu} e_{\nu} .
$$

Note that the rescaling of $\tilde{\kappa}$ corresponds to a rescaling of the Newton's constant $G$. Using (3.14), (3.22), (3.23) and (3.24) in the JT action (2.1), we find the following NR action in the $\varepsilon \rightarrow 0$ limit

$$
S_{N R J T}=\kappa \int d^{2} x \operatorname{det}(\tau e) \phi\left(\mathcal{R}^{N R}-2 \Lambda\right),
$$


where we have defined the NR Ricci scalar curvature

$$
\mathcal{R}^{N R} \equiv \stackrel{(-2)}{\mathcal{R}}=8 \tau^{[\mu} e^{\nu]} \partial_{\mu}\left(\tau^{[\alpha} e^{\beta]}\left(\partial_{\alpha} e_{\beta} e_{\nu}-\partial_{\alpha} m_{\beta} \tau_{\nu}\right)\right)
$$

This action leads to the second order equation

$$
\mathcal{R}^{N R}-2 \Lambda=0
$$

This defines the NR version of the relativistic JT equation (2.2a). Unlike second order gravitational actions in higher dimensions, (3.26) has no divergent terms. As we will see in the next section, the rescalings (3.24b) are compatible with the first-order formulation results. We will also show that the field equations of the second-order formalism are a submanifold of the field equations of the first order formalism.

\subsection{First order formulation}

The NR limit of JT gravity in the first-order formulation can be defined starting from a BF theory with gauge algebra $(A) \mathrm{dS}_{2} \times \mathbb{R}$. Thus we extend the $(\mathrm{A}) \mathrm{dS}_{2}$ symmetry (2.5) by including an Abelian generator $\tilde{Y}$ and consider the invariant bilinear form

$$
\langle\tilde{J}, \tilde{J}\rangle=\tilde{\gamma}_{0}, \quad\left\langle\tilde{P}_{a}, \tilde{P}_{b}\right\rangle=-\tilde{\gamma}_{0} \tilde{\Lambda} \eta_{a b}, \quad\langle\tilde{Y}, \tilde{Y}\rangle=-\tilde{\gamma}_{0} \tilde{\Lambda}
$$

The NR contraction follows from defining NR generators $H, P, J$ and $M$ as

$$
\begin{aligned}
\tilde{P}_{0} & =\frac{\varepsilon}{2} H+\frac{1}{\varepsilon} M, \\
\tilde{P}_{1} & =P
\end{aligned}
$$

$$
\begin{aligned}
\tilde{J} & =\frac{1}{\varepsilon} G, \\
\tilde{Y} & =\frac{\varepsilon}{2} H-\frac{1}{\varepsilon} M .
\end{aligned}
$$

Inverting this relation, we find

$$
\begin{aligned}
H & =\frac{1}{\varepsilon}\left(\tilde{P}_{0}+\tilde{Y}\right), \\
P & =\tilde{P}_{1},
\end{aligned}
$$

$$
\begin{aligned}
G & =\varepsilon \tilde{J}, \\
M & =\frac{\varepsilon}{2}\left(\tilde{P}_{0}-\tilde{Y}\right) .
\end{aligned}
$$

Using the commutation relations (2.5), and defining the NR cosmological constant $\Lambda$ as

$$
\Lambda=\frac{1}{\varepsilon^{2}} \tilde{\Lambda},
$$

we find, in the limit $\varepsilon \rightarrow 0$, the centrally Extended Newton-Hooke algebra [31, 32] in two space-time dimensions:

$$
[G, H]=P, \quad[G, P]=M, \quad[H, P]=-\Lambda G .
$$

Using the invariant tensor on $(\mathrm{A}) \mathrm{dS}_{2}$ (2.6) together with (3.29), and defining

$$
\gamma_{0}=\varepsilon^{2} \tilde{\gamma}_{0},
$$


the contraction (3.30) yields the following NR invariant bilinear form

$$
\langle G, G\rangle=\gamma_{0}, \quad\langle H, M\rangle=\gamma_{0} \Lambda, \quad\langle P, P\rangle=-\gamma_{0} \Lambda
$$

which is non-degenerate for $\gamma_{0} \neq 0$. We next consider the NR limit. of the JT action in first-order formulation (2.10). With this aim we consider a one-form connection taking values on $(\mathrm{A}) \mathrm{dS}_{2} \times \mathbb{R}$ algebra expressed in terms relativistic and NR generators [55]

$$
A=E^{a} \tilde{P}_{a}+\Omega \tilde{J}+X \tilde{Y}=\tau H+e P+\omega G+m M
$$

where, using (3.30), we find

$$
\begin{aligned}
& E^{0}=\frac{1}{\varepsilon} \tau+\frac{\varepsilon}{2} m, \\
& E^{1}=e \text {, } \\
& \Omega=\varepsilon \omega, \\
& X=\frac{1}{\varepsilon} \tau-\frac{\varepsilon}{2} m \text {. }
\end{aligned}
$$

Besides, we extend the definition of the scalar field (2.7) to take values on $(\mathrm{A}) \mathrm{dS}_{2} \times \mathbb{R}$ as well

$$
B=\Phi^{a} \tilde{P}_{a}+\Phi \tilde{J}+\Psi \tilde{Y}
$$

and define the NR scalar fields $\{\eta, \rho, \phi, \zeta\}$

$$
\begin{aligned}
& \Phi^{0}=\frac{1}{\varepsilon} \eta+\frac{\varepsilon}{2} \zeta, \\
& \Phi^{1}=\rho, \\
& \Phi=\varepsilon \phi, \\
& \Psi=\frac{1}{\varepsilon} \eta-\frac{\varepsilon}{2} \zeta .
\end{aligned}
$$

Using these definitions, the expansion of the relativistic BF action for the $(A) \mathrm{dS}_{2} \times \mathbb{R}$ algebra is

$$
\begin{aligned}
S[B, A] & =\tilde{\gamma}_{0} \int\left(\Phi R(\tilde{J})-\tilde{\Lambda} \Phi_{a} R^{a}(\tilde{P})\right)-\tilde{\gamma}_{0} \tilde{\Lambda} \int \Psi d \Psi \\
& =\varepsilon^{2} \tilde{\gamma}_{0} \int(\phi R(G)+\Lambda(\eta R(M)+\zeta R(H)-\rho R(P)))+\frac{\varepsilon^{4} \Lambda \tilde{\gamma}_{0}}{2} \int(\zeta \omega e-\phi m e-\rho \omega m),
\end{aligned}
$$

where we have used (3.32) and (3.29), and we have defined the NR curvature two-forms as

$$
\begin{aligned}
& R(H)=d \tau, \\
& R(P)=d e+\omega \tau,
\end{aligned}
$$$$
R(G)=d \omega-\Lambda \tau e,
$$

$$
R(M)=d m+\omega e .
$$

Using (3.34) and taking the limit $\varepsilon \rightarrow 0$, we obtain the NR two-dimensional gravity theory

$$
S=\gamma_{0} \int(\phi R(G)+\Lambda(\eta R(M)+\zeta R(H)-\rho R(P)))
$$


It is worth mentioning that this action can be alternatively obtained as a BF theory based on the Extended Newton-Hooke algebra (3.33), where the gauge connection $A$ and the $B$ field are given by

$$
\begin{aligned}
& A=\tau H+e P+\omega G+m M, \\
& B=\eta H+\rho P+\phi G+\eta M .
\end{aligned}
$$

In this case the curvature two form associated to $A$ takes the form

$$
R=R(H) H+R(P) P+R(G) G+R(M) M,
$$

with the components given in (3.41). It is straightforward to see that, using these definitions, the BF action leads exactly to (3.42) when using the NR invariant tensor (3.35).

The field equations coming from the action (3.42) when varying with respect to $\eta, \rho$, $\phi$ and $\zeta$ are given by

$$
\begin{aligned}
\delta \eta: & & R(H)=0, \\
\delta \rho: & & R(P)=0, \\
\delta \phi: & & R(G)=0, \\
\delta \zeta: & & R(M)=0,
\end{aligned}
$$

while varying (3.42) with respect to $\tau, e, \omega$ and $m$ leads to

$$
\begin{array}{rlrl}
\delta \tau & \text { r } & d \eta & =0, \\
\delta e: & d \rho+\omega \eta-\tau \phi & =0, \\
\delta \omega: & d \phi-\Lambda(\tau \rho-e \eta) & =0, \\
\delta m: & d \zeta+\omega \rho-e \phi & =0 .
\end{array}
$$

Note that equation (3.45a) implies

$$
d \tau=0 \quad \Longrightarrow \quad \tau=d \lambda,
$$

for some zero-form $\lambda$, therefore on-shell there is no torsion. This fact implies that the Newton-Cartan structure admits absolute time. Solving the field equations (3.46b) and $(3.46 \mathrm{~d})$ the spin-connection is given by (3.23). Therefore, the action (3.42) becomes

$$
S=\gamma_{0} \int \phi R(G)=-\frac{\gamma_{0}}{2} \int \operatorname{det}(\tau e) \phi\left(\mathcal{R}^{N R}-2 \Lambda\right) .
$$

This action matches with (3.26) identifying $\gamma_{0}=-2 \kappa$.

Since the spin connection has not been solved by its own field equation, this action is dynamically inequivalent to the first order action (3.42). This is in complete analogy to the relativistic case. 


\section{Carrollian Jackiw-Teitelboim gravity}

In this section, we work out the Carrollian limit of the JT gravity. We consider the Carroll contraction of the $(\mathrm{A}) \mathrm{dS}_{2} \times \mathbb{R}$ algebra. In order to do that we first interchange in $P_{0} \leftrightarrow P_{1}$, and $H \leftrightarrow P$ in (3.30), which gives

$$
\begin{aligned}
& \tilde{P}_{0}=H, \\
& \tilde{P}_{1}=\frac{\varepsilon}{2} P+\frac{1}{\varepsilon} M, \\
& \tilde{J}=\frac{1}{\varepsilon} G, \\
& \tilde{Y}=\frac{\varepsilon}{2} P-\frac{1}{\varepsilon} M .
\end{aligned}
$$

Secondly, taking the limit $\varepsilon \rightarrow 0$, and using the rescaling (3.32), we get

$$
[G, P]=H, \quad[G, H]=M, \quad[H, P]=-\Lambda G .
$$

We shall call this symmetry Extended Carroll $(A) d S_{2}$ algebra. One can see that these commutation relations follow from the Extended $\mathrm{NH}^{ \pm}$algebra (3.33) by interchanging $H$ and $P$ and changing the sign of the cosmological constant. This fact allows one to pass from Galilean to Carrollian symmetries. This relation is a generalisation in two dimensions of a more general duality between these two types of symmetries [48] previously found in the flat case.

This procedure defines the following dualities

$$
\begin{aligned}
& \text { Extended } \mathrm{NH}_{2}^{+} \leftrightarrow \text { Extended Carroll } \mathrm{AdS}_{2} \\
& \text { Extended } \mathrm{NH}_{2}^{-} \leftrightarrow \text { Extended Carroll } \mathrm{dS}_{2} .
\end{aligned}
$$

It is important to remark that in $1+1$ dimensions, the Carroll algebra (even without cosmological constant) admits the central extension $M$ given in (4.2) in the same way as its Galilean counterpart does. This situation is a unique feature of the two-dimensional case since, unlike the Galilean case, the Carroll algebra does not admit a non-trivial central extension in four dimensions.

\subsection{Second order formulation}

We start carrying out the Carrollian contraction in the second order formulation. To make contact with the Carrollian geometry we first write the relativistic zweibein in terms of the Carrollian zweibein gauge fields

$$
E_{\mu}^{0}=\tau_{\mu}, \quad E_{\mu}^{1}=\frac{1}{\varepsilon} e_{\mu}+\frac{\varepsilon}{2} m_{\mu} .
$$

The completeness relations $E_{a}^{\mu} E_{\nu}^{a}=\delta_{\nu}^{\mu}$, and $E_{b}^{\mu} E_{\mu}^{a}=\delta_{b}^{a}$ imply the inverse relativistic zweibein

$$
E_{0}^{\mu}=\tau^{\mu}-\frac{\varepsilon^{2}}{2} e^{\mu} \tau^{\nu} m_{\nu}, \quad E_{1}^{\mu}=\varepsilon e^{\mu}-\frac{\varepsilon^{3}}{2} e^{\mu} e^{\nu} m_{\nu}
$$

In analogy with (4.1), these expressions can be obtained by interchanging $E^{0}$ and $E^{1}$, and interchanging $\tau$ and $e$ in (3.7). The gauge fields $\tau, e$, and $m$ verifying the completeness relation (3.10). 
From the zweibein we can read the metric expansion

$$
g_{\mu \nu}=\frac{1}{\varepsilon^{2}} \stackrel{(2)}{g}_{\mu \nu}+\stackrel{(0)}{g}_{\mu \nu},
$$

where

$$
\stackrel{(2)}{g}_{\mu \nu}=e_{\mu} e_{\nu}, \quad \stackrel{(0)}{g}_{\mu \nu}=-\tau_{\mu} \tau_{\nu}+e_{(\mu} m_{\nu)} .
$$

Using the expression of the inverse zweibein (4.5), we find the following form of the inverse metric

$$
g^{\mu \nu}=-\tau^{\mu} \tau^{\nu}+\varepsilon^{2}\left(h^{\mu \nu}+\tau^{(\mu} e^{\nu)} \tau^{\sigma} m_{\sigma}\right)-\varepsilon^{4}\left(h^{\mu \nu} e^{\sigma} m_{\sigma}+\frac{1}{4} h^{\mu \nu} \tau^{\sigma} \tau^{\rho} m_{\sigma} m_{\rho}\right) .
$$

This has the general form

$$
g^{\mu \nu}=\stackrel{(0)}{g} \mu \nu+\varepsilon^{2} \stackrel{(-2)}{g} \mu \nu+\mathcal{O}\left(\varepsilon^{4}\right),
$$

where the components read

$$
\stackrel{(0)}{g}^{\mu \nu}=-\tau^{\mu} \tau^{\nu} \quad \stackrel{(-2)}{g} \mu \nu=h^{\mu \nu}+\tau^{(\mu} e^{\nu)} \tau^{\sigma} m_{\sigma},
$$

with the inverse degenerate spatial metric defined as $h^{\mu \nu}=e^{\mu} e^{\nu}$.

Note that (4.6) and (4.9) have the same form as the NR expansion given in the expression (3.1). However, in this case, the expansion parameter is defined as $\varepsilon=c$. Therefore, we will understand as Carrollian contraction the limit $\varepsilon \rightarrow 0$, which corresponds to the ultrarelativistic limit. With this consideration, the relations (3.2)-(3.6) also hold in this case.

The expansion (3.3) of the Carrollian affine connection can be obtained from the NR one (3.19) by interchanging the gauge fields $\tau$ and $e$. Similarly to the NR second-order formulation, the first non-vanishing term in the expansion of Ricci scalar (3.14) is given by

$$
\mathcal{R}^{\text {Carrollian }} \equiv \stackrel{(-2)}{\mathcal{R}}=4 e^{\mu} \tau^{\nu} \partial_{[\mu} \omega_{\nu]},
$$

where the Carrollian spin connection $\omega_{\mu}$ is

$$
\omega_{\mu}=2 e^{[\alpha} \tau^{\beta]}\left(\tau_{\mu} \partial_{\alpha} \tau_{\beta}-e_{\mu} \partial_{\alpha} m_{\beta}\right) .
$$

As before the Carrollian limit for the JT action (2.1), can be read off from (3.24) by interchanging $\tau$ and $e$. Finally, taking the limit $\varepsilon \rightarrow 0$, the Carrollian version of JT gravity is given by

$$
S_{\text {Carrollian JT }}=\kappa \int d^{2} x \operatorname{det}(\tau e) \phi\left(\mathcal{R}^{\text {Carrollian }}-2 \Lambda\right) .
$$

Notice that as in the NR case the divergent terms has been cancelled. This is a special property of two-dimensions.

\subsection{First order formulation}

In this section, we consider the Carrollian limit of the JT gravity in the first-order formalism. Let us consider a one-form connection taking values on $(\mathrm{A}) \mathrm{dS}_{2} \times \mathbb{R}$ algebra expressed in terms relativistic and Carrollian generators

$$
A=E^{a} \tilde{P}_{a}+\Omega \tilde{J}+X \tilde{Y}=e H+\tau P+\omega G+m M,
$$

where, using (4.1), we find 


$$
\begin{aligned}
& E^{0}=\tau, \\
& E^{1}=\frac{1}{\varepsilon} e+\frac{\varepsilon}{2} m, \\
& \Omega=\varepsilon \omega, \\
& X=\frac{1}{\varepsilon} e-\frac{\varepsilon}{2} m .
\end{aligned}
$$

Also we consider the scalar field to take values on $(\mathrm{A}) \mathrm{dS}_{2} \times \mathbb{R}$

$$
B=\Phi^{a} \tilde{P}_{a}+\Phi \tilde{J}+\Psi \tilde{Y},
$$

and define the Carrollian scalar fields $\{\eta, \rho, \phi, \zeta\}$ as

$$
\begin{aligned}
& \Phi^{0}=\eta, \\
& \Phi=\varepsilon \phi, \\
& \Phi^{1}=\frac{1}{\varepsilon} \rho+\frac{\varepsilon}{2} \zeta, \\
& \Psi=\frac{1}{\varepsilon} \rho-\frac{\varepsilon}{2} \zeta .
\end{aligned}
$$

These expressions can also be obtained from (4.17) by interchanging $\Phi^{0} \leftrightarrow \Phi^{1}$, and $\eta \leftrightarrow \rho$. Using these definitions, the relativistic BF action takes the form

$$
\begin{aligned}
S[B, A] & =\tilde{\gamma}_{0} \int\left(\Phi R(\tilde{J})-\tilde{\Lambda} \Phi_{a} R^{a}(\tilde{P})\right)-\tilde{\gamma}_{0} \tilde{\Lambda} \int \Psi d X \\
& =\varepsilon^{2} \tilde{\gamma}_{0} \int(\phi R(G)+\Lambda(\rho R(M)+\zeta R(P)-\eta R(H)))+\frac{\varepsilon^{4} \Lambda \tilde{\gamma}_{0}}{2} \int(\zeta \omega \tau-\phi m \tau-\eta \omega m),
\end{aligned}
$$

where we have used (3.32) and (3.29), and we have defined the Carrollian curvature twoforms as

$$
\begin{array}{lll}
R(H)=d \tau+\text { we, } & (4.19 \mathrm{a}) & R(G)=d \omega-\Lambda e \tau \\
R(P)=d e, & (4.19 \mathrm{~b}) & R(M)=d m+\omega \tau
\end{array}
$$

Using (3.34), the Carrollian limit is then obtained by taking $\varepsilon \rightarrow 0$, we find

$$
S=\gamma_{0} \int(\phi R(G)+\Lambda(\rho R(M)+\zeta R(P)-\eta R(H))) .
$$

This action can be alternatively obtained as a BF theory based on the Extended Carroll (A) $\mathrm{dS}_{2}$ algebra (4.2) and the bilinear form

$$
\langle G, G\rangle=\gamma_{0}, \quad\langle P, M\rangle=-\gamma_{0} \Lambda, \quad\langle H, H\rangle=\gamma_{0} \Lambda,
$$

which is non-degenerate for $\mu \neq 0$. The field equations coming from the action (4.20) when varying with respect to $\eta, \rho, \phi$, and $\zeta$ read

$$
\begin{array}{rlrl}
\delta \eta: & & R(H) & =0, \\
\delta \rho: & & R(M) & =0, \\
\delta \phi: & & R(G)=0, \\
\delta \zeta: & & R(P)=0,
\end{array}
$$


while varying (4.20) with respect to $\tau, e, \omega$ and $m$ leads to

$$
\begin{array}{rlrl}
e: & d \rho & =0, \\
\delta \tau: & & d \eta+\omega \rho-e \phi & =0, \\
\delta \omega: & d \phi+\Lambda(\tau \rho-e \eta) & =0, \\
\delta m: & d \zeta+\omega \eta-\tau \phi & =0 .
\end{array}
$$

Note that equation (4.22a) implies

$$
d e=0 \quad \Longrightarrow \quad e=d \lambda,
$$

for some zero-form $\lambda$. This defines an absolute one-dimensional space. Solving the field equations (4.23b) and (4.23d) yields (4.12). Finally, the action (4.20) becomes

$$
S=\gamma_{0} \int \phi R(G)=-\gamma_{0} \int \operatorname{det}(\tau e) \phi\left(\mathcal{R}^{\text {Carrollian }}-2 \Lambda\right),
$$

with the Carrollian curvature $\mathcal{R}^{\text {Carrollian }}$ given in (4.11).

\section{Relativistic boundary theory}

In the previous sections, we have considered the NR and Carrollian limits of JT gravity, focusing only on bulk dynamics and without paying attention to boundary terms. However, for the theory to have a well-defined variational principle as well as to define the holographic dual theory, we need to study the boundary dynamics. To address this problem, we consider general $\mathrm{BF}$ action of the form

$$
S[B, A]=\int_{\mathcal{M}}\langle B, F\rangle,
$$

where $F=d A+A \wedge A$. For now, we consider the zero-form $B$ and the one-form $A$ as taking values on a generic gauge Lie algebra $\mathcal{G}$ with a non-degenerate invariant bilinear form. In order to have a well defined variational principle, we follow the Regge-Teitelboim procedure [57] and supplement the action (5.1) with a boundary term

$$
S[B, A]=\int_{\mathcal{M}}\langle B, F\rangle+\int_{\partial \mathcal{M}} b,
$$

where $b$ is a one-form defined in such a way that, provided suitable boundary conditions for the gauge fields, the variation of the action does not drop boundary terms. A general variation of (5.2) leads to

$$
\delta S=\int_{\mathcal{M}}(\langle\delta B, F\rangle-\langle D B, \delta A\rangle)+\int_{\partial \mathcal{M}}(\delta b+\langle B, \delta A\rangle),
$$

where $D=d+[A, \cdot]$ is the covariant derivative. Considering the bulk coordinates $x^{\mu}=$ $(t, r)$ and the boundary defined at $r \rightarrow \infty$, the gauge fields can be decomposed as

$$
A=A_{t} d t+A_{r} d r, \quad b=b_{t} d t .
$$


Thus, the boundary term on the right hand side of (5.3) takes the form

$$
\int d t\left(\delta b_{t}+\left\langle B, \delta A_{t}\right\rangle\right)
$$

For this term to vanish, the boundary term $b$ must be such that

$$
\delta b_{t}=-\left.\left\langle B, \delta A_{t}\right\rangle\right|_{\partial \mathcal{M}} .
$$

A boundary condition on the field $B$ that allows to integrate the equation is [64]

$$
\left.B\right|_{\partial \mathcal{M}}=\left.k A_{t}\right|_{\partial \mathcal{M}} \quad \Longrightarrow \quad b_{t}=-\frac{k}{2}\left\langle A_{t}, A_{t}\right\rangle,
$$

with $k$ an arbitrary constant. On the other hand, the field equations coming from (5.3) imply that $B$ is covariantly constant, while $A$ is locally pure gauge, i.e. $A=g^{-1} d g$ with $g$ an element belonging to a gauge group $\mathcal{G}$. Furthermore, we consider the following gauge fixing condition [34]

$$
\partial_{t} A_{r}=0 \Longrightarrow g(t, r)=\mathrm{U}(t) b(r) .
$$

The gauge connection then takes the form

$$
A=b^{-1} d b+b^{-1} a b,
$$

where $a=U^{-1} d U$ only depends on the coordinate $t$. Evaluating the action (5.2) on this solution space, the theory is reduced to the boundary and the action reads

$$
S_{\text {bdy }}[U]=-\frac{k}{2} \int d t\left\langle U^{-1} U^{\prime}, U^{-1} U^{\prime}\right\rangle,
$$

where the prime stands for derivative with respect to $t$. An important step in our construction is that at this point we can consider the boundary target coordinate $t$ as a world-line parameter of a particle, and the connection $a$ as the pull-back of the left-invariant MaurerCartan (MC) form $\Omega$ on $\mathcal{G}$, i.e.

$$
a=\Omega^{\star},
$$

where $\Omega$ satisfies the MC equations

$$
d \Omega+\Omega \wedge \Omega=0 .
$$

Therefore, the boundary action (5.10) can be interpreted as the action of a particle moving on the group manifold of $\mathcal{G}$

$$
S_{\text {bdy }}[U]=-\frac{k}{2} \int d t\langle\Omega, \Omega\rangle^{\star} .
$$

Given a specific form of the connection (5.11), one can look for the residual gauge transformations

$$
\delta_{\lambda} a=d \lambda+[a, \lambda] .
$$


that preserve its form. The corresponding conserved charges can then be computed using the known result for BF theory $[58,59]$

$$
\delta Q\left[\lambda_{b u l k}\right]=-\int\left\langle\lambda_{b u l k}, \delta B\right\rangle d \tau_{E},
$$

where we have switched to Euclidean time $\tau_{E}$ in order to have a periodic coordinate. Imposing the boundary condition (5.7) for the field $B$ and using the usual decomposition $\lambda_{b u l k}=b(r)^{-1} \lambda(t) b(r)$, we find the expression

$$
\delta Q[\lambda]=-k \int\left\langle\lambda, \delta a_{t}\right\rangle d \tau_{E}
$$

Provided the boundary charges can be integrated, the charge algebra is obtained from the transformation law $[34,57]$

$$
\delta_{\lambda} Q[\bar{\lambda}]=\{Q[\bar{\lambda}], Q[\lambda]\}
$$

In the following, we will apply this procedure in the relativistic case and subsequently in the different NR and Carrollian scenarios.

\section{$5.1(\mathrm{~A}) \mathrm{dS}_{2}$ case}

As a first example we consider the $(\mathrm{A}) \mathrm{dS}_{2}$ symmetry and the derivation of the Schwarzian action $[4,7,8]$. The $\mathrm{AdS}_{2}$ and the $\mathrm{dS}_{2}$ cases can be treated in a unified way by going to the conformal basis, as both they are isomorphic to the $\mathfrak{s l}(2, \mathbb{R})$ algebra (see appendix A)

$$
[\tilde{\mathcal{H}}, \tilde{\mathcal{D}}]=\tilde{\mathcal{H}}, \quad[\tilde{\mathcal{K}}, \tilde{\mathcal{D}}]=-\tilde{\mathcal{K}}, \quad[\tilde{\mathcal{H}}, \tilde{\mathcal{K}}]=2 \tilde{\mathcal{D}}
$$

In this basis, the non-degenerate invariant bilinear form reads

$$
\langle\tilde{\mathcal{D}}, \tilde{\mathcal{D}}\rangle=\tilde{\gamma}_{0}, \quad\langle\tilde{\mathcal{H}}, \tilde{\mathcal{K}}\rangle=-2 \tilde{\gamma}_{0}
$$

Then, it is possible to locally parametrize an arbitrary group element $\tilde{U}$ as

$$
\tilde{U}=e^{\tilde{\mathcal{H}} \tilde{\mathcal{H}}} e^{\tilde{y} \tilde{\mathcal{K}}} e^{\tilde{u} \tilde{\mathcal{D}}}
$$

with $\tilde{\rho}, \tilde{y}$, and $\tilde{u}$ the group manifold coordinates. They will be the Goldstone fields when we take the pull-back to the world-line of the particle. To simplify the computation of the MC one-form $\tilde{\Omega}$, we use the following $2 \times 2$ representation of the $\mathfrak{s l}(2, \mathbb{R})$ algebra

$$
\tilde{\mathcal{H}}=\left(\begin{array}{ll}
0 & 0 \\
1 & 0
\end{array}\right), \quad \tilde{\mathcal{D}}=\frac{1}{2}\left(\begin{array}{cc}
1 & 0 \\
0 & -1
\end{array}\right), \quad \tilde{\mathcal{K}}=\left(\begin{array}{cc}
0 & -1 \\
0 & 0
\end{array}\right) .
$$

Locally we can parametrize the group element in (5.20) as

$$
\tilde{U}=\left(\begin{array}{cc}
e^{\frac{\tilde{u}}{2}} & -\tilde{y} e^{-\frac{\tilde{u}}{2}} \\
\tilde{\rho} e^{\frac{\tilde{u}}{2}} & e^{-\frac{\tilde{u}}{2}}(1-\tilde{\rho} \tilde{y})
\end{array}\right)
$$


The MC one-form $\tilde{\Omega}=\tilde{U}^{-1} d \tilde{U}$ is given in a matrix representation by

$$
\tilde{\Omega}=\tilde{\Omega}_{\tilde{\mathcal{H}}} \tilde{\mathcal{H}}+\tilde{\Omega}_{\tilde{\mathcal{D}}} \tilde{\mathcal{D}}+\tilde{\Omega}_{\tilde{\mathcal{K}}} \tilde{\mathcal{K}}=\left(\begin{array}{cc}
\frac{1}{2} \tilde{\Omega}_{\tilde{\mathcal{D}}}-\tilde{\Omega}_{\tilde{\mathcal{K}}} \\
\tilde{\Omega}_{\tilde{\mathcal{H}}}-\frac{1}{2} \tilde{\Omega}_{\tilde{\mathcal{D}}}
\end{array}\right),
$$

where we have defined the one-forms,

$$
\tilde{\Omega}_{\tilde{\mathcal{H}}}=e^{\tilde{u}} d \tilde{\rho}, \quad \tilde{\Omega}_{\tilde{\mathcal{K}}}=e^{-\tilde{u}}\left(\tilde{y}^{2} d \tilde{\rho}+d \tilde{y}\right), \quad \tilde{\Omega}_{\tilde{\mathcal{D}}}=d \tilde{u}+2 \tilde{y} d \tilde{\rho} .
$$

The MC one-forms can be used to construct the boundary action (5.13),

$$
S[U]=-\frac{k}{2} \int d t\langle\tilde{\Omega}, \tilde{\Omega}\rangle^{\star}=-\frac{k \tilde{\gamma}_{0}}{2} \int d t\left(4 \tilde{\rho}^{\prime}\left(\tilde{y} \tilde{u}^{\prime}-\tilde{y}^{\prime}\right)+\tilde{u}^{\prime 2}\right)
$$

where prime denotes derivative with respect to the world-line parameter $t$. This model is invariant under the global symmetries

$$
\delta \tilde{\rho}=\tilde{\alpha}+\tilde{\beta} \tilde{\rho}-\tilde{\gamma} \tilde{\rho}^{2}, \quad \delta \tilde{y}=(2 \tilde{\gamma} \tilde{\rho}-\tilde{\beta}) \tilde{y}-\tilde{\gamma}, \quad \delta \tilde{u}=2 \tilde{\gamma} \tilde{\rho}-\tilde{\beta},
$$

where the symmetry parameters can be grouped as

$$
\tilde{\epsilon}=\tilde{\alpha} \tilde{\mathcal{H}}+\tilde{\beta} \tilde{\mathcal{D}}+\tilde{\gamma} \tilde{\mathcal{K}}=\left(\begin{array}{cc}
\frac{1}{2} \tilde{\beta} & -\tilde{\gamma} \\
\tilde{\alpha} & -\frac{1}{2} \tilde{\beta}
\end{array}\right) .
$$

We identify in (5.26) the transformation for the Goldstone field $\tilde{\rho}$ as the infinitesimal version of a $\operatorname{SL}(2, R)$ Möbius transformation.

We can reduce the number of Goldstone fields in the action by setting some MC forms to zero or defining (invariant) covariant relations among them. This procedure is also known as the inverse Higgs mechanism (IHM) [30]. In the following, we present an example of this method. ${ }^{8}$ We consider the constraints

$$
\tilde{\Omega}_{\tilde{\mathcal{H}}}=\mu, \quad \tilde{\Omega}_{\tilde{\mathcal{D}}}=0,
$$

with $\mu$ a constant. This allows us to express $\tilde{y}$ and $\tilde{u}$ in terms of $\tilde{\rho}$ as follows,

$$
\tilde{u}=\log \left(\frac{\mu}{\tilde{\rho}^{\prime}}\right), \quad \tilde{y}=-\frac{\tilde{u}^{\prime}}{2 \tilde{\rho}^{\prime}},
$$

for some constant $\mu$. Let us evaluate the MC form on the constraints (5.28)

$$
\left.a \equiv \tilde{\Omega}^{\star}\right|_{I H M}=\mu \tilde{\mathcal{H}}+\mathcal{L}(\tilde{\rho}) \tilde{\mathcal{K}}=\left(\begin{array}{cc}
0 & -\mathcal{L}(\tilde{\rho}) \\
\mu & 0
\end{array}\right),
$$

where we have introduced the function $\mathcal{L}(\tilde{\rho}) \equiv \frac{1}{2 \mu} \operatorname{Sch}(\tilde{\rho}, t)$, with

$$
\operatorname{Sch}(\tilde{\rho}, t)=\frac{\tilde{\rho}^{\prime \prime \prime}}{\tilde{\rho}^{\prime}}-\frac{3}{2}\left(\frac{\tilde{\rho}^{\prime \prime}}{\tilde{\rho}^{\prime}}\right)^{2}
$$

\footnotetext{
${ }^{8}$ We do not attempt to classify all possible IHM constraints.
} 
which is the Schwarzian derivative. In this case, the IHM is equivalent to a Drinfeld-Sokolov reduction $[62,63]$. Plugging (5.29) into the action (5.25), we find

$$
S[\tilde{\rho}]=k \tilde{\gamma}_{0} \int d t \operatorname{Sch}(\tilde{\rho}, t),
$$

which corresponds to the Schwarzian mechanical model invariant under a global $\mathrm{SL}(2, R)$ transformation

$$
\tilde{\rho}_{\epsilon}=\frac{a \tilde{\rho}+b}{c \tilde{\rho}+d}, \quad a b-c d=1, \quad \Longrightarrow \quad S\left[\tilde{\rho}_{\epsilon}\right]=S[\tilde{\rho}] .
$$

This result shows that it is possible to obtain the Schwarzian action using the IHM. ${ }^{9}$

In order to obtain the gauge field in the bulk, we can introduce a radial dependence by performing a gauge transformation with the group element

$$
b=\left(\begin{array}{cc}
e^{r / 2 \ell} & 0 \\
0 & e^{-r / 2 \ell}
\end{array}\right)
$$

this leads to the following form for the connection (5.9)

$$
A=b^{-1} d b+b^{-1} \Omega^{\star} b=\left(\begin{array}{cc}
\frac{d r}{2 \ell}+\frac{1}{2} \tilde{\Omega}_{\tilde{\mathcal{D}}}^{\star} & -e^{-r / \ell} \tilde{\Omega}_{\tilde{\mathcal{K}}}^{\star} \\
e^{r / \ell} \tilde{\Omega}_{\tilde{\mathcal{H}}}^{\star} & -\frac{d r}{2 \ell}-\frac{1}{2} \tilde{\Omega}_{\tilde{\mathcal{D}}}^{\star}
\end{array}\right),
$$

where we have used (5.11) that implements the pull-back of the $\mathrm{MC}$ forms to the boundary coordinate $t$. Choosing $\mu=\frac{1}{2}$, we find the gauge field given in [64], namely

$$
A=\left(\begin{array}{cc}
\frac{d r}{2 \ell} & 0 \\
0 & -\frac{d r}{2 \ell}
\end{array}\right)+\frac{d t}{2}\left(\begin{array}{cc}
0 & -2 \operatorname{Sch}(\tilde{\rho}, t) e^{-r / \ell} \\
e^{r / \ell} & 0
\end{array}\right) .
$$

This shows that IHM relations can be interpreted as boundary conditions on the BF connection $A$. Using the change of variables that relates $\mathfrak{s l}(2, \mathbb{R})$ and $(\mathrm{A}) \mathrm{dS}_{2}$ algebras (see appendix A)

$$
\tilde{\mathcal{D}}=\ell \tilde{P}_{1}, \quad \tilde{\mathcal{H}}=\ell \tilde{P}_{0}+\tilde{J}, \quad \tilde{\mathcal{K}}=\ell \tilde{P}_{0}-\tilde{J},
$$

we can write down the explicit form for the gravitational gauge fields in (2.8). In the $\mathrm{AdS}_{2}$ case we find

$$
\begin{aligned}
E^{0} & =\ell\left(\frac{1}{2} e^{r / \ell}+\operatorname{Sch}(\tilde{\rho}, t) e^{-r / \ell}\right) d t, \\
E^{1} & =d r \\
\Omega & =\left(\frac{1}{2} e^{r / \ell}-\operatorname{Sch}(\tilde{\rho}, t) e^{-r / \ell}\right) d t .
\end{aligned}
$$

\footnotetext{
${ }^{9}$ The Schwarzian can also be obtained by integrating out the gauge transformations of the particle model with variables $x^{\mu}, \lambda$ and Lagrangian $L=\frac{1}{2} \dot{x}^{2}-\frac{1}{2} \lambda x^{2}$, see [60, 61].
} 
In order to write down the zweibein and the spin connection for the $\mathrm{dS}_{2}$ case, we interchange the coordinates $t$ and $r / \ell$. This is due to the fact that the de-Sitter space has a boundary at time-like infinity. In this particular case, the prime denotes derivative with respect to $r$. We find

$$
\begin{aligned}
E^{0} & =\ell d t \\
E^{1} & =\left(\frac{1}{2} e^{t}+\operatorname{Sch}(\tilde{\rho}, r / \ell) e^{-t}\right) d r \\
\Omega & =\frac{1}{\ell}\left(\frac{1}{2} e^{t}-\operatorname{Sch}(\tilde{\rho}, r / \ell) e^{-t}\right) d r .
\end{aligned}
$$

From the expression of the zweibeine, we find the following the space-time metrics

$$
\begin{aligned}
d s_{A d S}^{2} & =-\ell^{2}\left(\frac{1}{4} e^{2 r / \ell}+\operatorname{Sch}(\tilde{\rho}, t)+e^{-2 r / \ell} \operatorname{Sch}(\tilde{\rho}, t)^{2}\right) d t^{2}+d r^{2}, \\
d s_{d S}^{2} & =-\ell^{2} d t^{2}+\left(\frac{1}{4} e^{2 t}+\operatorname{Sch}(\tilde{\rho}, r / \ell)+e^{-2 t} \operatorname{Sch}(\tilde{\rho}, r / \ell)^{2}\right) d r^{2} .
\end{aligned}
$$

If we consider the leading term of (5.40) for $r \rightarrow \infty$ we recover the boundary conditions of [64].

The asymptotic symmetry is the residual symmetry that leaves the conditions (5.30) invariant. These conditions restrict the gauge parameter

$$
\lambda=\lambda_{0} \tilde{\mathcal{H}}+\lambda_{1} \tilde{\mathcal{K}}+\lambda_{2} \tilde{\mathcal{D}}=\left(\begin{array}{cc}
\frac{1}{2} \lambda_{2} & -\lambda_{1} \\
\lambda_{0} & -\frac{1}{2} \lambda_{2}
\end{array}\right)
$$

The resulting gauge parameter is given by

$$
\lambda=\lambda_{0} \tilde{\mathcal{H}}+\left(\mathcal{L} \lambda_{0}+\frac{1}{2} \lambda_{0}^{\prime \prime}\right) \tilde{\mathcal{K}}-\lambda_{0}^{\prime} \tilde{\mathcal{D}}
$$

whereas the transformation law of the function $\mathcal{L}$ leads to

$$
\delta \mathcal{L}=\lambda_{0} \mathcal{L}^{\prime}+2 \mathcal{L} \lambda_{0}^{\prime}+\frac{1}{2} \lambda_{0}^{\prime \prime \prime},
$$

which is the transformation of a stress tensor under infinitesimal conformal transformations. Using (5.16), the associated charge reads

$$
Q\left[\lambda_{0}\right]=2 \tilde{\gamma}_{0} k \int \lambda_{0} \mathcal{L} d \tau_{E}
$$

Then, the Poisson bracket (5.17), leads to the Virasoro algebra ${ }^{10}$

$$
\left\{Q\left[\lambda_{0}\right], Q\left[\bar{\lambda}_{0}\right]\right\}=Q\left[\left[\lambda_{0}, \bar{\lambda}_{0}\right]\right]+\tilde{\gamma}_{0} k \int \lambda_{0} \bar{\lambda}_{0}^{\prime \prime \prime} d \tau_{E},
$$

\footnotetext{
${ }^{10}$ Here prime denotes derivative with respect to the periodic coordinate $\tau_{E}$.
} 
where $[x, y]=x y^{\prime}-y x^{\prime}$ stands for the Lie bracket, and we have changed to Euclidean time in (5.44). Introducing the Fourier modes

$$
\mathcal{L}_{m} \equiv Q\left[e^{i m \tau_{E}}\right], \quad m \in \mathbb{Z},
$$

the Poisson algebra (5.46) takes the usual form

$$
\left\{\mathcal{L}_{m}, \mathcal{L}_{n}\right\}=(m-n) \mathcal{L}_{m+n}+\frac{c_{1}}{12} m^{3} \delta_{m+n, 0}, \quad c_{1}=24 \pi \tilde{\gamma}_{0} k
$$

where we have considered the following representation for the Kronecker delta $\delta_{m n}=$ $\frac{1}{2 \pi} \int e^{i(m-n) \tau_{E}} d \tau_{E}$.

\subsection{Derivation of the boundary action in second order formalism}

In the second order formulation, the JT gravity action (2.1) has to be supplemented with an appropriate boundary term in order for the variational principle to be well-defined. The result action reads

$$
S_{\mathrm{JT}}=\tilde{\kappa} \int_{\mathcal{M}} d^{2} x \sqrt{-g} \Phi(\mathcal{R}-2 \tilde{\Lambda})+2 \tilde{\kappa} \int_{\partial \mathcal{M}} d t \sqrt{-h} \Phi_{b}\left(K-K_{0}\right),
$$

where $t$ correspond to the boundary local coordinate on $\partial \mathcal{M}, h$ is the determinant of the induced one-dimensional metric on the boundary, $\Phi_{b}$ is the boundary value of the scalar field $\Phi, K$ the trace of the extrinsic curvature, and $K_{0}$ the trace of the extrinsic curvature of the boundary computed in AdS spacetime. The expression for $K$ is given by

$$
K=\frac{1}{2} g^{\mu \nu} P^{\alpha}{ }_{\mu} P^{\beta}{ }_{\nu} £_{\hat{N}} g_{\alpha \beta},
$$

where we have defined the projector $P^{\alpha}{ }_{\mu} \equiv \delta_{\mu}^{\alpha}-\hat{N}^{\alpha} \hat{N}_{\mu}$, and $£_{\hat{N}}$ corresponding to the Lie derivative along the unit normal vector $\hat{N}$ to the boundary curve.

Now, our goal is to evaluate the JT action (5.49) on the (locally) AdS space-time metric (5.40). Because this metric has constant negative curvature $\mathcal{R}=-2 / \ell^{2}$, then the first term in (5.49) vanishes. We only need to evaluate the boundary term. To this end, we notice that the boundary condition (5.7) and the boundary form of (5.36) can be translated into boundary conditions for the metric and the scalar field that enter in the JT action. Indeed, using the change of basis (5.37) one finds

$$
A_{t}=k\left(\frac{1}{2} e^{r / \ell}-\operatorname{Sch}(\rho, t) e^{-r / \ell}\right) \tilde{J}+k \ell\left(\frac{1}{2} e^{r / \ell}+\operatorname{Sch}(\rho, t) e^{-r / \ell}\right) \tilde{P}_{0},
$$

so that the boundary condition (5.7) leads to

$$
\left.B\right|_{\partial M}=\frac{k}{2} e^{r_{0} / \ell}\left(\tilde{J}+\ell \tilde{P}_{0}\right)
$$

and therefore, from eq. (3.38), we find

$$
\Phi_{b}=\frac{k}{2 e^{-r_{0} / \ell}},
$$


where $r_{0}$ is some large fixed value of the radial coordinate $r$, and the boundary is defined by $r_{0} \rightarrow \infty$. On the other hand, the unit normal vector is given by

$$
\hat{N}=\frac{\partial}{\partial r} \text {. }
$$

We also need the induced metric on the boundary, which can be obtained evaluating (5.40) on $r_{0}$, finding

$$
\left.d s_{\mathrm{AdS}}^{2}\right|_{r=r_{0}}=-\ell^{2}\left(\frac{1}{2} e^{r_{0} / \ell}+\operatorname{Sch}(\tilde{\rho}, t) e^{-r_{0} / \ell}\right)^{2} d t^{2},
$$

with determinant, at leading order in $r_{0}$, given by

$$
h=-\frac{\ell^{2}}{\left(2 e^{-r_{0} / \ell}\right)^{2}}+\mathcal{O}\left(r_{0}^{-4}\right) .
$$

The expressions (5.53) and (5.56) define boundary conditions in the second order formulation [64]. ${ }^{11}$ One can now explicitly evaluate the trace of the extrinsic curvature (5.50) to find

$$
K=\left.\frac{1}{2} g^{t t} \partial_{r} g_{t t}\right|_{r=r_{0}}=\frac{1}{\ell}\left(1-4 e^{-2 r_{0} / \ell} \operatorname{Sch}(\tilde{\rho}, t)\right)+\mathcal{O}\left(r_{0}^{-4}\right) .
$$

Plugging (5.53), (5.56) and (5.57) into (5.49), and setting $K_{0}=1 / \ell$ the action at leading order in $r_{0}$ becomes

$$
S_{\mathrm{JT}}=-2 \tilde{\kappa} k \int d t \operatorname{Sch}(\tilde{\rho}, t),
$$

in agreement with (5.32) when we identify $\tilde{\gamma}_{0}=-2 \tilde{\kappa}$, as previously done with the bulk action in (2.19).

\section{$5.3(A) \mathrm{dS}_{2} \times \mathbb{R}$ case}

We turn now our attention to the $(\mathrm{A}) \mathrm{dS}_{2} \times \mathbb{R}$ algebra where the gauge connection is given by

$$
A=E^{a} \tilde{P}_{a}+\Omega \tilde{J}+X \tilde{Y} .
$$

In the conformal basis (see appendix A), this algebra corresponds to $\mathfrak{s l}(2, \mathbb{R}) \times \mathbb{R}$, with its relations given in (5.18). The non-degenerate invariant bilinear form is given (3.29) which in the conformal basis can be written as

$$
\langle\tilde{\mathcal{D}}, \tilde{\mathcal{D}}\rangle=\tilde{\gamma}_{0}, \quad\langle\tilde{\mathcal{H}}, \tilde{\mathcal{K}}\rangle=-2 \tilde{\gamma}_{0}, \quad\langle\tilde{\mathcal{Y}}, \tilde{\mathcal{Y}}\rangle=\tilde{\gamma}_{1}
$$

where $\tilde{\mathcal{Y}} \equiv \tilde{Y}$. Now, let us consider the local parametrisation of a group element $\tilde{U}$ associated to (5.18) as follows

$$
\tilde{U}=e^{\tilde{s} \tilde{\mathcal{Y}}} e^{\tilde{\rho} \tilde{\mathcal{H}}} e^{\tilde{y} \tilde{\mathcal{K}}} e^{\tilde{u} \tilde{\mathcal{D}}}
$$

\footnotetext{
${ }^{11}$ Note that defining a parameter $\epsilon=2 e^{-} r_{0} / \ell$, the expressions (5.53) and (5.56) are the Lorentzian version of the boundary conditions given in $[9,64]$

$$
\Phi_{b}=\frac{k}{\epsilon}, \quad h=-\frac{\ell^{2}}{\epsilon^{2}}, \quad \epsilon \rightarrow 0 .
$$
}


where $\tilde{s}, \tilde{\rho}, \tilde{y}$, and $\tilde{u}$ are the group manifold coordinates. Using the matrix representation (5.21), and considering $\tilde{\mathcal{Y}}=\mathbb{1}_{2 \times 2}$, the group element in (5.61) can be written as

$$
\tilde{U}=\left(\begin{array}{cc}
e^{\tilde{s}+\frac{\tilde{u}}{2}} & -\tilde{y} e^{\tilde{s}-\frac{\tilde{u}}{2}} \\
\tilde{\rho} e^{\tilde{s}+\frac{\tilde{u}}{2}} & e^{\tilde{s}-\frac{\tilde{u}}{2}}(1-\tilde{\rho} \tilde{y})
\end{array}\right)
$$

The MC one-form $\tilde{\Omega}=\tilde{U}^{-1} d \tilde{U}$ is given by

$$
\tilde{\Omega}=\tilde{\Omega}_{\tilde{\mathcal{H}}} \tilde{\mathcal{H}}+\tilde{\Omega}_{\tilde{\mathcal{D}}} \tilde{\mathcal{D}}+\tilde{\Omega}_{\tilde{\mathcal{K}}} \tilde{\mathcal{K}}+\tilde{\Omega}_{\tilde{\mathcal{Y}}} \tilde{\mathcal{Y}}
$$

where the one-forms have the form

$$
\begin{array}{lll}
\tilde{\Omega}_{\tilde{\mathcal{H}}}=e^{\tilde{u}} d \tilde{\rho}, & (5.64 \mathrm{a}) & \tilde{\Omega}_{\tilde{\mathcal{D}}}=d \tilde{u}+2 \tilde{y} d \tilde{\rho} \\
\tilde{\Omega}_{\tilde{\mathcal{K}}}=e^{-\tilde{u}}\left(\tilde{y}^{2} d \tilde{\rho}+d \tilde{y}\right), & (5.64 \mathrm{~b}) & \tilde{\Omega}_{\tilde{\mathcal{Y}}}=d \tilde{s}
\end{array}
$$

The MC one-forms can be used to construct the boundary action (5.13)

$$
S[\tilde{\rho}, \tilde{y}, \tilde{u}, \tilde{s}]=-\frac{k}{2} \int d t(\langle\tilde{\Omega}, \tilde{\Omega}\rangle)^{\star}=-\frac{k}{2} \int d t\left(\tilde{\gamma}_{0}\left(4 \tilde{\rho}^{\prime}\left(\tilde{y} \tilde{u}^{\prime}-\tilde{y}^{\prime}\right)+\tilde{u}^{\prime 2}\right)+\tilde{\gamma}_{1} \tilde{s}^{\prime 2}\right)
$$

which is invariant under the following global symmetries

$$
\begin{aligned}
\delta \tilde{\rho} & =\tilde{\beta}-\tilde{\alpha} \tilde{\rho}+\tilde{\gamma}^{2} \tilde{\rho}^{2}, \\
\delta \tilde{u} & =\tilde{\alpha}-2 \tilde{\gamma} \tilde{\rho}, \\
\delta \tilde{y} & =\tilde{\gamma}+\tilde{y}(\tilde{\alpha}-2 \tilde{\gamma} \tilde{\rho}), \\
\delta \tilde{s} & =\tilde{\theta},
\end{aligned}
$$

associated to the infinitesimal symmetry parameter

$$
\tilde{\epsilon}=\tilde{\alpha} \tilde{\mathcal{H}}+\tilde{\beta} \tilde{\mathcal{D}}+\tilde{\gamma} \tilde{\mathcal{K}}+\tilde{\theta} \tilde{\mathcal{Y}}=\left(\begin{array}{cc}
\frac{1}{2} \tilde{\beta}+\tilde{\theta} & -\tilde{\gamma} \\
\tilde{\alpha} & -\frac{1}{2} \tilde{\beta}+\tilde{\theta}
\end{array}\right)
$$

In the following, we consider the following inverse Higgs constraints [27]

$$
\tilde{\Omega}_{\tilde{\mathcal{H}}}=\mu \tilde{\Omega}_{\tilde{\mathcal{Y}}}, \quad \tilde{\Omega}_{\tilde{\mathcal{D}}}=-2 \nu \tilde{\Omega}_{\tilde{\mathcal{Y}}},
$$

where $\mu$ and $\nu$ are arbitrary constants. We can express $\tilde{y}$ and $\tilde{u}$ in terms of the independent Goldstone fields $\tilde{\rho}$ and $\tilde{s}$ as

$$
\tilde{y}=-\frac{2 \nu \tilde{s}^{\prime}+\tilde{u}^{\prime}}{2 \tilde{\rho}^{\prime}}, \quad \tilde{u}=\log \left(\frac{\mu \tilde{s}^{\prime}}{\tilde{\rho}^{\prime}}\right) .
$$

In this case, the MC one-form (5.63) reduces to

$$
\left.\tilde{a} \equiv \tilde{\Omega}^{\star}\right|_{I H M}=\mu \tilde{s}^{\prime} \tilde{\mathcal{H}}+\tilde{s}^{\prime} \tilde{\mathcal{Y}}-2 \nu \tilde{s}^{\prime} \tilde{\mathcal{D}}+\frac{1}{2 \mu \tilde{s}^{\prime}}\left(\operatorname{Sch}(\tilde{\rho}, t)-\operatorname{Sch}(\tilde{s}, t)+\nu^{2} \tilde{s}^{2}\right) \tilde{\mathcal{K}}
$$


and therefore the action (5.65) is reduced to

$$
S[\tilde{\rho}, \tilde{s}]=-\frac{k}{2} \int d t\left(-2 \tilde{\gamma}_{0}(\operatorname{Sch}(\tilde{\rho}, t)-\operatorname{Sch}(\tilde{s}, t))+\tilde{\gamma}_{1} \tilde{s}^{2}\right) .
$$

Note that, if we impose the condition $\tilde{s}-t=0$ in (5.71), we recover the Schwarzian action (5.32).

To construct the gauge field in the bulk, we can introduce a radial dependence by performing a gauge transformation (5.9) with the group element (5.34). Using the matrix representation (5.21), leads to the following form for the connection (5.59)

$$
A=\left(\begin{array}{cc}
\frac{d r}{2 \ell} & 0 \\
0 & -\frac{d r}{2 \ell}
\end{array}\right)+d t\left(\begin{array}{cc}
(1-\nu) \tilde{s}^{\prime}-e^{-r / \ell}\left[\frac{1}{2 \mu \tilde{s}^{\prime}}(\operatorname{Sch}(\tilde{\rho}, t)-\operatorname{Sch}(\tilde{s}, t))+\frac{\nu^{2}}{\mu} \tilde{s}^{\prime}\right] \\
\mu e^{r / \ell} \tilde{s}^{\prime} & (1+\nu) \tilde{s}^{\prime}
\end{array}\right),
$$

from which we can read off the zweibein and spin connection. In the $\mathrm{AdS}_{2}$ case they read

$$
\begin{aligned}
E^{0} & =\ell\left(\mu s^{\prime} e^{r / \ell}+\left[\frac{1}{2 \mu \tilde{s}^{\prime}}(\operatorname{Sch}(\tilde{\rho}, t)-\operatorname{Sch}(\tilde{s}, t))+\frac{\nu^{2}}{\mu} \tilde{s}^{\prime}\right] e^{-r / \ell}\right) d t, \\
E^{1} & =d r \\
\Omega & =\left(\mu s^{\prime} e^{r / \ell}-\left[\frac{1}{2 \mu \tilde{s}^{\prime}}(\operatorname{Sch}(\tilde{\rho}, t)-\operatorname{Sch}(\tilde{s}, t))+\frac{\nu^{2}}{\mu} \tilde{s}^{\prime}\right] e^{-r}\right) d t,
\end{aligned}
$$

whereas, for the $\mathrm{dS}_{2}$ case we find

$$
\begin{aligned}
E^{0} & =\ell d t, \\
E^{1} & =\left(\mu s^{\prime} e^{t}+\left[\frac{1}{2 \mu \tilde{s}^{\prime}}(\operatorname{Sch}(\tilde{\rho}, r / \ell)-\operatorname{Sch}(\tilde{s}, r \ell))+\frac{\nu^{2}}{\mu} \tilde{s}^{\prime}\right] e^{-t}\right) d r, \\
\Omega & =\frac{1}{\ell}\left(\mu s^{\prime} e^{t}-\left[\frac{1}{2 \mu \tilde{s}^{\prime}}(\operatorname{Sch}(\tilde{\rho}, r / \ell)-\operatorname{Sch}(\tilde{s}, r / \ell))+\frac{\nu^{2}}{\mu} \tilde{s}^{\prime}\right] e^{-t}\right) d r,
\end{aligned}
$$

As done in (5.39), we have interchanged the coordinates $t$ and $r / \ell$ in the $\mathrm{dS}_{2}$ case (5.74), since the boundary of the de-Sitter space is at time-like infinity (in this case the prime denotes derivative with respect to the radial coordinate). From these expressions, we can construct the space-time metrics

$$
\begin{array}{r}
d s_{A d S}^{2}=-\ell^{2}\left(\mu^{2} s^{\prime 2} e^{2 r / \ell}-\left[(\operatorname{Sch}(\tilde{\rho}, t)-\operatorname{Sch}(\tilde{s}, t))+2 \nu^{2} \tilde{s}^{\prime 2}\right]\right. \\
\left.+e^{-2 r / \ell}\left[\frac{1}{2 \mu \tilde{s}^{\prime}}(\operatorname{Sch}(\tilde{\rho}, t)-\operatorname{Sch}(\tilde{s}, t))+\frac{\nu^{2}}{\mu} \tilde{s}^{\prime}\right]^{2}\right) d t^{2}+d r^{2} \\
d s_{d S}^{2}=-\ell^{2} d t^{2}+\left(\mu^{2} s^{\prime 2} e^{2 r / \ell}-\left[(\operatorname{Sch}(\tilde{\rho}, r / \ell)-\operatorname{Sch}(\tilde{s}, r / \ell))+2 \nu^{2} \tilde{s}^{2}\right]\right. \\
\left.+e^{-2 r / \ell}\left[\frac{1}{2 \mu \tilde{s}^{\prime}}(\operatorname{Sch}(\tilde{\rho}, r / \ell)-\operatorname{Sch}(\tilde{s}, r / \ell))+\frac{\nu^{2}}{\mu} \tilde{s}^{\prime}\right]^{2}\right) d r^{2} .
\end{array}
$$


Analogously to the previous section, one can ask what are the asymptotic symmetries associated with the theory defined by (5.71). For simplicity let us consider the case $\nu=0$, and rewrite the boundary field (5.70) as follows

$$
\tilde{a}=\left(\mu \mathcal{\mathcal { H }}+\frac{1}{\mu} \frac{\mathcal{L}}{\mathcal{T}} \tilde{\mathcal{K}}+\mathcal{T} \tilde{\mathcal{Y}}\right) d t
$$

where we have defined the functions

$$
\mathcal{T}(t) \equiv \tilde{s}^{\prime}, \quad \mathcal{L}(t) \equiv \frac{1}{2}(\operatorname{Sch}(\tilde{\rho}, t)-\operatorname{Sch}(\tilde{s}, t)) .
$$

The asymptotic symmetries correspond to the set of gauge transformations (5.14) preserving the functional form of (5.77). Considering a gauge parameter of the form

$$
\lambda=\lambda_{0} \tilde{\mathcal{H}}+\lambda_{1} \tilde{\mathcal{K}}+\lambda_{2} \tilde{\mathcal{D}}+\lambda_{3} \tilde{\mathcal{Y}}
$$

one can solve (5.14) to find

$$
\begin{aligned}
& \lambda_{0}=\mu \mathcal{T} \epsilon \\
& \lambda_{1}=\frac{1}{\mu \mathcal{T}}\left[\frac{1}{2}\left(\epsilon^{\prime}+\frac{\mathcal{T}^{\prime}}{\mathcal{T}} \epsilon-\chi^{\prime}-\frac{\mathcal{T}^{\prime}}{\mathcal{T}} \chi\right)^{\prime}+\mathcal{L} \epsilon\right], \\
& \lambda_{2}=-\epsilon^{\prime}-\frac{\mathcal{T}^{\prime}}{\mathcal{T}} \epsilon+\chi^{\prime}+\frac{\mathcal{T}^{\prime}}{\mathcal{T}} \chi, \\
& \lambda_{3}=\mathcal{T} \chi .
\end{aligned}
$$

The functions $\mathcal{T}$ and $\mathcal{L}$ transform under the asymptotic symmetries as

$$
\begin{aligned}
& \delta \mathcal{L}=\epsilon \mathcal{L}^{\prime}+2 \mathcal{L} \epsilon^{\prime}-\frac{\mathcal{T}^{\prime}}{2 \mathcal{T}}\left(\epsilon^{\prime}+\frac{\mathcal{T}^{\prime}}{\mathcal{T}} \epsilon-\chi^{\prime}-\frac{\mathcal{T}^{\prime}}{\mathcal{T}} \chi\right)^{\prime}+\frac{1}{2}\left(\epsilon^{\prime}+\frac{\mathcal{T}^{\prime}}{\mathcal{T}} \epsilon-\chi^{\prime}-\frac{\mathcal{T}^{\prime}}{\mathcal{T}} \chi\right)^{\prime \prime} \\
& \delta \mathcal{T}=\chi^{\prime} \mathcal{T}+\chi \mathcal{T}^{\prime}
\end{aligned}
$$

Using the explicit form of the gauge parameters, the charge can be integrated to give

$$
Q[\epsilon, \chi]=L[\epsilon]+T[\chi],
$$

where we have defined

$$
\begin{aligned}
& L[\epsilon]=k \tilde{\gamma}_{0} \int \epsilon\left(2 \mathcal{L}+\left(\frac{\mathcal{T}^{\prime}}{\mathcal{T}}\right)^{\prime}-\frac{1}{2}\left(\frac{\mathcal{T}^{\prime}}{\mathcal{T}}\right)^{2}\right) d \tau_{E}, \\
& T[\chi]=-\frac{k}{2} \int \chi\left(\tilde{\gamma}_{1} \mathcal{T}^{2}+\tilde{\gamma}_{0}\left(\frac{\mathcal{T}^{\prime}}{\mathcal{T}}\right)^{\prime}-\tilde{\gamma}_{0}\left(\frac{\mathcal{T}^{\prime}}{\mathcal{T}}\right)^{2}\right) d \tau_{E} .
\end{aligned}
$$

The Poisson algebra of these charges can be computed using (5.17), which yields two Virasoro algebras

$$
\begin{gathered}
\left\{L\left[\epsilon_{1}\right], L\left[\epsilon_{2}\right]\right\}=-L\left[\left[\epsilon_{1}, \epsilon_{2}\right]\right]-k \tilde{\gamma}_{0} \int \epsilon_{1}^{\prime} \epsilon_{2}^{\prime \prime} d \tau_{E}, \\
\left\{T\left[\chi_{1}\right], T\left[\chi_{2}\right]\right\}=-T\left[\left[\chi_{1}, \chi_{2}\right]\right]+k \tilde{\gamma}_{0} \int \chi_{1}^{\prime} \chi_{2}^{\prime \prime} d \tau_{E} .
\end{gathered}
$$


Introducing the Fourier modes

$$
\mathcal{L}_{m}=L\left[e^{i m \tau_{E}}\right], \quad \mathcal{T}_{m}=T\left[e^{i m \tau_{E}}\right],
$$

and changing to Euclidean time in the variations (5.81), the corresponding Poisson algebra leads to two Virasoro algebras

$$
\begin{gathered}
\left\{\mathcal{L}_{m}, \mathcal{L}_{n}\right\}=(m-n) \mathcal{L}_{n+m}-2 \pi k \tilde{\gamma}_{0} m^{3} \delta_{m+n, 0}, \\
\left\{\mathcal{T}_{m}, \mathcal{T}_{n}\right\}=(m-n) \mathcal{T}_{n+m}+2 \pi k \tilde{\gamma}_{0} m^{3} \delta_{m+n, 0}
\end{gathered}
$$

The central terms have the same form but with opposite signs, which is compatible with the form of the double Schwarzian boundary action (5.71).

Finally, it is worth to mention that from the constraint $\tilde{\Omega}_{\tilde{\mathcal{H}}}=\mu \tilde{\Omega}_{\tilde{\mathcal{Y}}}$ we can express $\tilde{\rho}^{\prime}$ in terms of $\tilde{s}^{\prime}$ and $\tilde{u}$,

$$
\rho^{\prime}=e^{-u} s^{\prime}
$$

resulting in the action

$$
S[\tilde{u}, \tilde{s}]=\int d t\left(\tilde{\gamma}_{0}\left(\tilde{u}^{\prime 2}+2 \tilde{u}^{\prime \prime}-\frac{2 \tilde{s}^{\prime \prime} \tilde{u}^{\prime}}{\tilde{s}^{\prime}}\right)+\tilde{\gamma}_{1} \tilde{s}^{2}\right) .
$$

In the next section, we will show that this last model admits a finite NR limit on its fields, which leads to a NR conformal model invariant under a NR conformal symmetry isomorphic to the Extended Newton-Hooke algebra.

\section{Non-relativistic boundary theory}

In this section, we construct the boundary theory associated with the NR JT gravity and compute the asymptotic symmetries and their associated conserved charges. We show how these results can be extended to the Carrollian case.

\subsection{Non-relativistic limit of the $\operatorname{SL}(2, \mathbb{R}) \times \mathbb{R}$ boundary action}

Here, we derive a NR limit of the relativistic model in (5.90). In order to do that, we consider the following redefinition of the $\mathrm{SL}(2, \mathbb{R}) \times \mathbb{R}$ generators

$$
\begin{aligned}
& \tilde{\mathcal{D}}=\frac{1}{2} \mathcal{D}+\frac{1}{\varepsilon^{2}} \mathcal{Z}, \\
& \tilde{\mathcal{Y}}=\frac{1}{2} \mathcal{D}-\frac{1}{\varepsilon^{2}} \mathcal{Z},
\end{aligned}
$$

$$
\begin{aligned}
\tilde{\mathcal{H}} & =\frac{1}{\varepsilon} \mathcal{H}, \\
\tilde{\mathcal{K}} & =\frac{1}{\varepsilon} \mathcal{K},
\end{aligned}
$$

whose inverse reads

$$
\begin{aligned}
& \mathcal{D}=\tilde{\mathcal{D}}+\tilde{\mathcal{Y}}, \\
& \mathcal{Z}=\frac{\varepsilon^{2}}{2}(\tilde{\mathcal{D}}-\tilde{\mathcal{Y}}),
\end{aligned}
$$

$$
\mathcal{H}=\varepsilon \tilde{\mathcal{H}}
$$$$
\mathcal{K}=\varepsilon \tilde{\mathcal{K}} .
$$

Taking the limit $\varepsilon \rightarrow 0$, we get

$$
[\mathcal{H}, \mathcal{D}]=\mathcal{H}, \quad[\mathcal{K}, \mathcal{D}]=-\mathcal{K}, \quad[\mathcal{H}, \mathcal{K}]=2 \mathcal{Z},
$$


which we name Extended Galilean conformal (EGC) algebra in 1D. This algebra is isomorphic to the $\mathrm{NH}^{+}$algebra. In fact, using the change of basis

$$
\mathcal{D}=\ell H, \quad \mathcal{H}=\ell P+G, \quad \mathcal{K}=\ell P-G, \quad \mathcal{Z}=\ell M
$$

we find the commutation relations (3.33) with cosmological constant $\Lambda=1 / \ell^{2}$

$$
[G, H]=P, \quad[G, P]=M, \quad[H, P]=-\frac{1}{\ell^{2}} G .
$$

Using the transformation (6.1) and its inverse (6.2), one can relate relativistic Goldstone fields in terms of NR ones as

$$
\begin{array}{lll}
\tilde{u}=u+\frac{\varepsilon^{2}}{2} s, & (6.6 \mathrm{a}) & \tilde{y}=\varepsilon y, \\
\tilde{\rho}=\varepsilon \rho, & (6.6 \mathrm{~b}) & \tilde{s}=u-\frac{\varepsilon^{2}}{2} s .
\end{array}
$$

Therefore, applying the field transformations (6.6a) and (6.6d) on the action (5.90), we find

$$
S[u, s]=2 \varepsilon^{2} \tilde{\gamma}_{0} \int d t\left(s^{\prime \prime}+s^{\prime}\left(u^{\prime}-\frac{u^{\prime \prime}}{u^{\prime}}\right)\right)+\mathcal{O}\left(\varepsilon^{4}\right),
$$

where we have set $\tilde{\gamma}_{1}=-\tilde{\gamma}_{0}$ in order to cancel a divergent term. Now, defining $\tilde{\gamma}_{0} \equiv \gamma_{0} / \varepsilon^{2}$, in the limit $\varepsilon \rightarrow 0$, the NR model becomes

$$
S[u, s]=2 \gamma_{0} \int d t\left(s^{\prime \prime}+s^{\prime}\left(u^{\prime}-\frac{u^{\prime \prime}}{u^{\prime}}\right)\right) .
$$

We shall refer to this action as the Non-Relativistic Schwarzian. Note that this boundary action appears at order in $\varepsilon^{2}$ in the same way that the NR gravity theory on the bulk does in (3.40). It is important to remark that if we perform the NR limit of the boundary action when expressed in terms of Schwarzians (5.71), the divergent term is not canceled.

In order to obtain the $\mathrm{NH}^{-}$algebra, we consider the following redefinition of the $\mathfrak{s l}(2, \mathbb{R}) \times \mathbb{R}$ generators

$$
\begin{aligned}
& \tilde{\mathcal{H}}=\frac{1}{2} \mathcal{D}+\frac{1}{2 \varepsilon}(\mathcal{H}-\mathcal{K})+\frac{1}{\varepsilon^{2}} \mathcal{Z}, \\
& \tilde{\mathcal{D}}=\frac{1}{2 \varepsilon}(\mathcal{H}+\mathcal{K}), \\
& \tilde{\mathcal{K}}=\frac{1}{2} \mathcal{D}-\frac{1}{2 \varepsilon}(\mathcal{H}-\mathcal{K})+\frac{1}{\varepsilon^{2}} \mathcal{Z},
\end{aligned}
$$

Inverting these relations leads to

$$
\begin{aligned}
& \mathcal{H}=\varepsilon\left[\tilde{\mathcal{D}}+\frac{1}{2}(\tilde{\mathcal{H}}-\tilde{\mathcal{K}})\right], \\
& \mathcal{D}=\frac{1}{2}(\tilde{\mathcal{H}}+\tilde{\mathcal{K}})+\tilde{\mathcal{Y}}, \\
& \mathcal{K}=\varepsilon\left[\tilde{\mathcal{D}}-\frac{1}{2}(\tilde{\mathcal{H}}-\tilde{\mathcal{K}})\right], \\
& \mathcal{Z}=\frac{\varepsilon^{2}}{2}\left[\frac{1}{2}(\tilde{\mathcal{H}}+\tilde{\mathcal{K}})-\tilde{\mathcal{Y}}\right] .
\end{aligned}
$$

In the limit $\varepsilon \rightarrow 0$, we find the following NR contraction of $\mathfrak{s l}(2, \mathbb{R}) \times \mathbb{R}$

$$
[\hat{\mathcal{H}}, \hat{\mathcal{D}}]=\hat{\mathcal{K}}, \quad[\hat{\mathcal{K}}, \hat{\mathcal{D}}]=-\hat{\mathcal{H}}, \quad[\hat{\mathcal{H}}, \hat{\mathcal{K}}]=2 \hat{\mathcal{Z}},
$$


which will be referred to as Twisted Extended Galilean Conformal algebra in 1D. By defining the following change of basis

$$
\hat{\mathcal{D}}=\ell H, \quad \hat{\mathcal{H}}=\ell P+G, \quad \hat{\mathcal{K}}=\ell P-G, \quad \hat{\mathcal{Z}}=\ell M,
$$

we find the $\mathrm{NH}_{2}^{-}$algebra (3.33) with $\Lambda=-1 / \ell^{2}$

$$
[G, H]=P, \quad[G, P]=M, \quad[H, P]=\frac{1}{\ell^{2}} G .
$$

It is important to note that the NR symmetries (6.3) and (6.11) are related by the complex change of basis

$$
\begin{array}{lll}
\hat{\mathcal{D}}=-i \mathcal{D}, & (6.14 \mathrm{a}) & \hat{\mathcal{H}}=\mathcal{H}+\mathcal{K}, \\
\hat{\mathcal{Z}}=2 i \mathcal{Z}, & (6.14 \mathrm{~b}) & \hat{\mathcal{K}}=-i(\mathcal{H}-\mathcal{K}) .
\end{array}
$$

Therefore, one can consider the analogue change of variables between the relativistic Goldstone fields $\tilde{s}, \tilde{u}$ and the ones of the Extended $\mathrm{NH}^{-}$algebra $\hat{s}, \hat{u}$, i.e.

$$
\tilde{u}=-i \hat{u}+\frac{i \varepsilon^{2}}{2} \hat{s}, \quad \tilde{s}=-i \hat{u}-\frac{i \varepsilon^{2}}{2} \hat{s} .
$$

Applying these field transformations into (5.90), we get

$$
S[\hat{u}, \hat{s}]=2 i \varepsilon^{2} \tilde{\gamma}_{0} \int d t\left(\hat{s}^{\prime \prime}-\hat{s}^{\prime}\left(i \hat{u}^{\prime}+\frac{\hat{u}^{\prime \prime}}{\hat{u}^{\prime}}\right)\right)+\mathcal{O}\left(\varepsilon^{4}\right)
$$

again setting $\tilde{\gamma}_{1}=-\tilde{\gamma}_{0}$, and taking the definition $\tilde{\gamma}_{0} \equiv \gamma_{0} / \varepsilon^{2}$, in the limit $\varepsilon \rightarrow 0$, we get the complexified version of NR Schwarzian (6.8), namely

$$
S[\hat{u}, \hat{s}]=2 i \gamma_{0} \int d t\left(\hat{s}^{\prime \prime}-\hat{s}^{\prime}\left(i \hat{u}^{\prime}+\frac{\hat{u}^{\prime \prime}}{\hat{u}^{\prime}}\right)\right) .
$$

This action is closely related to the one obtained in [18]. In the following, we will derive these same actions using an IHM.

\subsection{Extended Newton-Hooke ${ }_{2}^{+}$case}

Following the steps outlined in section 5, we construct the MC one-form associated to the Extended Galilean conformal algebra (6.3) by considering a local parametrisation of a group element $U$ as

$$
U=e^{s \mathcal{Z}} e^{\rho \mathcal{H}} e^{y \mathcal{K}} e^{u \mathcal{D}},
$$

where $s, \rho, y$, and $u$ correspond to the set of NR coordinates of the group manifold. We consider a faithful $3 \times 3$ matrix representation of (6.3) given by

$$
\mathcal{Z}=\left(\begin{array}{lll}
0 & 0 & \frac{1}{2} \\
0 & 0 & 0 \\
0 & 0 & 0
\end{array}\right), \quad \mathcal{H}=\left(\begin{array}{lll}
0 & 1 & 0 \\
0 & 0 & 0 \\
0 & 0 & 0
\end{array}\right), \quad \mathcal{K}=\left(\begin{array}{lll}
0 & 0 & 0 \\
0 & 0 & 1 \\
0 & 0 & 0
\end{array}\right), \quad \mathcal{D}=\left(\begin{array}{lll}
0 & 0 & 0 \\
0 & 1 & 0 \\
0 & 0 & 0
\end{array}\right)
$$


so that the group element $U$ reads

$$
U=\left(\begin{array}{ccc}
1 & \rho e^{u} & \frac{s}{2}+\rho y \\
0 & e^{u} & y \\
0 & 0 & 1
\end{array}\right)
$$

The left-invariant MC one-form is then given by

$$
\Omega=U^{-1} d U=\Omega_{\mathcal{Z}} \mathcal{Z}+\Omega_{\mathcal{H}} \mathcal{H}+\Omega_{\mathcal{K}} \mathcal{K}+\Omega_{\mathcal{D}} \mathcal{D},
$$

with components

$$
\Omega_{\mathcal{Z}}=d s+2 y d \rho, \quad \Omega_{\mathcal{H}}=e^{u} d \rho, \quad \Omega_{\mathcal{K}}=e^{-u} d y, \quad \Omega_{\mathcal{D}}=d u .
$$

The invariant bilinear form in this case reads (6.3)

$$
\langle\mathcal{D}, \mathcal{D}\rangle=\gamma_{1}, \quad\langle\mathcal{D}, \mathcal{Z}\rangle=\gamma_{0}, \quad\langle\mathcal{H}, \mathcal{K}\rangle=-2 \gamma_{0},
$$

where $\gamma_{0}$ and $\gamma_{1}$ are arbitrary constants. This bilinear form is non-degenerate for $\gamma_{0} \neq 0$. Using these expressions, the boundary action (5.13) takes the form

$$
S[s, \rho, y, u]=\int d t\left(\gamma_{1} u^{\prime 2}+2 \gamma_{0}\left(u^{\prime}\left(s^{\prime}+2 y \rho^{\prime}\right)-2 \rho^{\prime} y^{\prime}\right)\right) .
$$

and is invariant under the following global symmetry transformations

$$
\delta s=\theta-2 \gamma \rho, \quad \delta \rho=\beta-\alpha \rho, \quad \delta y=\gamma+\alpha y, \quad \delta u=\alpha,
$$

where the infinitesimal parameters can be grouped as $\epsilon=\theta \mathcal{Z}+\beta \mathcal{H}+\gamma \mathcal{K}+\alpha \mathcal{D}$.

In order to reduce the number of Goldstone fields, we will impose a particular IHM constraint. Let us consider the constraints

$$
\Omega_{\mathcal{Z}}=0, \quad \Omega_{\mathcal{H}}=\alpha,
$$

with $\alpha$ a constant. The boundary theory (6.24) then reduces to

$$
S[u, s]=\int d t\left(\gamma_{1} u^{\prime 2}+2 \gamma_{0}\left(s^{\prime \prime}+s^{\prime} u^{\prime}\right)\right) .
$$

Note that, performing the change of variables

$$
u=v-\log \left(v^{\prime}\right),
$$

and setting $\gamma_{1}=0$ in the bilinear form (6.23), we recover the action (6.8)

$$
S[v, s]=2 \gamma_{0} \int d t\left(s^{\prime \prime}+s^{\prime}\left(v^{\prime}-\frac{v^{\prime \prime}}{v^{\prime}}\right)\right) .
$$

In this case the boundary connection takes the form

$$
a=\left.\Omega^{*}\right|_{I H M}=(\alpha \mathcal{H}+\mathcal{T}(t) \mathcal{D}+\mathcal{L}(t) \mathcal{K}) d t=\left(\begin{array}{ccc}
0 & \alpha & 0 \\
0 & \mathcal{T}(t) & \mathcal{L}(t) \\
0 & 0 & 0
\end{array}\right),
$$


where we have defined the functions

$$
\mathcal{T}(t) \equiv v^{\prime}-\frac{v^{\prime \prime}}{v^{\prime}}, \quad \mathcal{L}(t) \equiv \frac{1}{2 \alpha v^{\prime}}\left(s^{\prime}\left(v^{\prime \prime}-v^{\prime 2}\right)-s^{\prime \prime} v^{\prime}\right) .
$$

The gauge transformations preserving these boundary conditions are obtained by solving (5.14), which gives

$$
\begin{aligned}
& \lambda_{\mathcal{Z}}=-2 \alpha \chi, \\
& \lambda_{\mathcal{H}}=\alpha \sigma, \\
& \lambda_{\mathcal{K}}=\chi^{\prime}+\mathcal{L} \sigma, \\
& \lambda_{\mathcal{D}}=\sigma^{\prime}+\mathcal{T} \sigma,
\end{aligned}
$$

whereas the variations of $\mathcal{L}$ and $\mathcal{T}$ read

$$
\begin{aligned}
& \delta \mathcal{L}(t)=\sigma \mathcal{L}^{\prime}+2 \mathcal{L} \sigma^{\prime}+\mathcal{T} \chi^{\prime}-\chi^{\prime \prime} \\
& \delta \mathcal{T}(t)=\sigma^{\prime} \mathcal{T}+\sigma \mathcal{T}^{\prime}-\sigma^{\prime \prime}
\end{aligned}
$$

The charge (5.16) can be integrated, leading to

$$
Q[\sigma, \chi]=L[\sigma]+T[\chi],
$$

where

$$
\begin{aligned}
& L[\sigma]=2 \alpha \gamma_{0} k \int \sigma \mathcal{L} d \tau_{E}, \\
& T[\chi]=2 \alpha \gamma_{0} k \int \chi \mathcal{T} d \tau_{E} .
\end{aligned}
$$

The Poisson algebra (5.17) in this case takes the form of the Twisted Warped Virasoro algebra $[17,18,65]$, which after expanding in Fourier modes

$$
\mathcal{L}_{m} \equiv L\left[e^{i m \tau_{E}}\right], \quad \mathcal{T}_{m} \equiv T\left[e^{i m \tau_{E}}\right],
$$

has the form

$$
\begin{aligned}
& \left\{\mathcal{L}_{m}, \mathcal{L}_{n}\right\}=(m-n) \mathcal{L}_{n+m} \\
& \left\{\mathcal{L}_{m}, \mathcal{T}_{n}\right\}=-n \mathcal{T}_{m+n}+4 \pi \alpha \gamma_{0} m \delta_{n+m, 0} \\
& \left\{\mathcal{T}_{m}, \mathcal{T}_{n}\right\}=0
\end{aligned}
$$

We can introduce the dependence on a radial coordinate $r$ by applying a gauge transformation with an $r$-dependent group element of the form [18]

$$
b=e^{-\frac{r}{\ell} \mathcal{H}}=\left(\begin{array}{ccc}
1 & -r / \ell & 0 \\
0 & 1 & 0 \\
0 & 0 & 1
\end{array}\right) .
$$

This leads to

$$
A=\left(-\frac{d r}{\ell}+\left(\frac{r}{\ell} \mathcal{T}(t)+\alpha\right) d t\right) \mathcal{H}+\left(\mathcal{L}(t) \mathcal{K}+\mathcal{T}(t) \mathcal{D}+\frac{2 r}{\ell} \mathcal{L}(t) \mathcal{Z}\right) d t
$$


Using the change of basis (6.4) we can find the asymptotic form of the NR gravitational gauge fields

$$
\begin{aligned}
\tau & =\ell \mathcal{T} d t \\
e & =-d r+(r \mathcal{T}+\ell \alpha+\ell \mathcal{L}) d t, \\
\omega & =-\frac{d r}{\ell}+\left(\frac{r}{\ell} \mathcal{T}+\alpha-\mathcal{L}\right) d t, \\
m & =2 r \mathcal{L} d t
\end{aligned}
$$

which satisfy the field equations (3.45). Using (6.41) and the completeness relations (3.10) one fiends that the inverse dreibein takes the simple form

$$
e^{\mu}=(0,-1)
$$

Thus the Newton-Cartan structure is defined by the inverse spatial metric

$$
h^{\mu \nu}=\left(\begin{array}{ll}
0 & 0 \\
0 & 1
\end{array}\right),
$$

which is degenerate with null vector

$$
\tau_{\mu}=(\ell \mathcal{T}, 0)
$$

Similarly, one can construct the spatial metric

$$
h_{\mu \nu}=(r \mathcal{T}+\ell \alpha+\ell \mathcal{L})^{2} d t^{2}-2(r \mathcal{T}+\ell \alpha+\ell \mathcal{L}) d r d t+d r^{2},
$$

whose null vector is given by

$$
\tau^{\mu}=\frac{1}{\ell \mathcal{T}}(1, r \mathcal{T}+\ell \alpha+\ell \mathcal{L})
$$

\subsection{Extended Newton-Hooke $e_{2}^{-}$case}

Now we consider the boundary action associated with the Twisted Extended Conformal algebra (6.11), isomorphic to the $\mathrm{NH}_{2}^{-}$symmetry. The $\mathrm{NR}$ analysis in the case of negative cosmological constant can be done in the same way as the previous case by using the Extended Galilean conformal algebra (6.3) and the complex change of basis (6.1). This relates the coordinates of the coset (6.18) to new coordinates $\{\hat{s}, \hat{\rho}, \hat{y}, \hat{u}\}$ associated to the algebra (6.11),

$$
\begin{aligned}
& \rho=\frac{1}{\sqrt{2}}(\hat{\rho}-i \hat{y}), \\
& y=\frac{1}{\sqrt{2}}(\hat{\rho}+i \hat{y}), \\
& u=-i \hat{u}, \\
& s=i \hat{s} .
\end{aligned}
$$

Applying these transformations directly to the reduced action (6.24), we find

$$
S[\hat{u}, \hat{s}]=i \int d \tau\left(i \gamma_{1} \hat{u}^{2}+2 \gamma_{0}\left(\hat{s}^{\prime \prime}-\hat{s}^{\prime}\left(i \hat{u}^{\prime}+\frac{\hat{u}^{\prime \prime}}{\hat{u}^{\prime}}\right)\right)\right) .
$$


This action is invariant under the global symmetries

$$
\delta \hat{s}=\tilde{\theta}+i(\hat{\beta}+i \hat{\gamma})(\hat{\rho}-i \hat{y}), \quad \delta \hat{\rho}=\hat{\beta}+\hat{\alpha} \hat{y}, \quad \delta \hat{y}=\hat{\gamma}-\hat{\alpha} \hat{\rho}, \quad \delta \hat{u}=\hat{\alpha},
$$

with symmetry parameter $\hat{\epsilon}=\hat{\theta} \hat{\mathcal{Z}}+\hat{\beta} \hat{\mathcal{H}}+\hat{\gamma} \hat{\mathcal{K}}+\hat{\alpha} \hat{\mathcal{D}}$. The action (6.48) matches the model found in [18] as the flat limit of the Schwarzian action.

To perform the asymptotic analysis, we can use (6.30) and the change of basis (6.1) to write the boundary connection as

$$
\left.a \equiv \Omega^{*}\right|_{I H M}=\left(\frac{1}{2}(\alpha+\mathcal{L}(t)) \hat{\mathcal{H}}+i \mathcal{T}(t) \hat{\mathcal{D}}+\frac{i}{2}(\alpha-\mathcal{L}(t)) \hat{\mathcal{K}}\right) d t
$$

Then, the same analysis as the previous section leads to the variations (6.33) for the functions $\mathcal{L}(t)$ and $\mathcal{T}(t)$ and the asymptotic symmetry (6.38).

\subsection{Extended Carroll (A) $\mathrm{dS}_{2}$ case}

Due to the duality (4.3), the Extended Conformal Galilean algebra (6.3) and its twisted version (6.11) are isomorphic to Carrollian symmetries. Indeed starting from (6.3) and using the change of basis

$$
\mathcal{D}=\ell P, \quad \mathcal{H}=\ell H+G, \quad \mathcal{K}=\ell H-G, \quad \mathcal{Z}=\ell M,
$$

leads to the Carroll $\mathrm{AdS}_{2}$ algebra

$$
[G, P]=H, \quad[G, H]=M, \quad[H, P]=\frac{1}{\ell^{2}} G .
$$

Similarly, applying the change of basis

$$
\mathcal{D}=\ell P, \quad \mathcal{H}=\ell H+G, \quad \mathcal{K}=\ell H-G, \quad \mathcal{Z}=\ell M,
$$

to the algebra (6.11) yields the Carroll $\mathrm{dS}_{2}$ algebra

$$
[G, P]=H, \quad[G, H]=M, \quad[H, P]=-\frac{1}{\ell^{2}} G .
$$

This means that the boundary actions (6.27) and (6.48) can also be interpreted as a boundary action of Carrollian JT gravity. Thus, the asymptotic symmetry analysis done in section 6.2 also holds here and leads to the Warped Virasoro symmetry (6.38).

Similarly to the NR case, the change of variables (6.51) for the Carroll $\mathrm{AdS}_{2}$ case can be used to define a Carrollian geometry. Indeed, the asymptotic form of the Carrollian gravitational gauge fields read

$$
\begin{aligned}
e & =\ell \mathcal{T} d t \\
\tau & =-d r+(r \mathcal{T}+\ell \alpha+\ell \mathcal{L}) d t, \\
\omega & =-\frac{d r}{\ell}+\left(\frac{r}{\ell} \mathcal{T}+\alpha-\mathcal{L}\right) d t, \\
m & =2 r \mathcal{L} d t .
\end{aligned}
$$


In this case, the Carrollian geometric structure is described by the temporal metric

$$
\tau_{\mu \nu} \equiv-\tau_{\mu} \tau_{\nu}=-(r \mathcal{T}+\ell \alpha+\ell \mathcal{L})^{2} d t^{2}+2(r \mathcal{T}+\ell \alpha+\ell \mathcal{L}) d r d t+d r^{2},
$$

which is degenerate and has a null vector given by

$$
e^{\mu}=\frac{1}{\ell \mathcal{T}}(1, r \mathcal{T}+\ell \alpha+\ell \mathcal{L})
$$

A similar construction for the Carroll $\mathrm{dS}_{2}$ can be done by means of the change of variables (6.53).

\section{Conclusions and outlook}

We have constructed the NR and Carrollian limits of JT gravity theory and the corresponding boundary actions. The analysis in the bulk is done first by considering the NR and Carrollian limits of a BF action with gauge algebra (A) $\mathrm{dS}_{2} \times \mathbb{R}$. Next, we have constructed $\mathrm{BF}$ theories with gauge algebras given by $\mathrm{NH}^{ \pm}$and Carroll $(\mathrm{A}) \mathrm{dS}_{2}$. The Carrollian analysis is easily done using the duality (4.3) between NR and Carrollian symmetries. The NR and Carrollian second order theories in the bulk are constructed by considering a suitable expansion of the metric tensor up to order $1 / c^{2}$ and $c^{2}$, respectively. The actions are such that no divergent terms appear in the expansion if Newton's constant is properly rescaled. This property is due to the fact that in two dimensions there are no divergent terms in the expansion of the Ricci scalar. This is a remarkably property that is in high contrast with its analogue in three and four space-time dimensions [43-46].

The boundary actions associated with the NR and Carroll JT gravities have been obtained using the method of non-linear realisations and IHM. In particular, we have shown that this reproduces the Drinfeld-Sokolov reduction of $\operatorname{SL}(2, \mathbb{R})[62,63]$, which has been previously used in [64] to define boundary conditions of JT gravity in analogy to the three-dimensional gravity case [66]. The procedure has been generalised to the case of the $\mathrm{SL}(2, \mathbb{R}) \times \mathbb{R}$ algebra and the conformal descriptions of $\mathrm{NH}_{2}^{ \pm}$and Extended Carroll $(\mathrm{A}) \mathrm{dS}_{2}$. This leads to the NR Schwarzian action (6.8) and its complexification (6.17). We have also derived the corresponding conserved charges and the asymptotic symmetry of these theories, which is given by the twisted Warped Virasoro algebra [17, 18, 65]. Using the explicit form of the boundary gauge fields, we have reconstructed the fields in the bulk, which define Newton-Cartan [35-40] and Carrollian structures [41, 42].

Further studies can be done, for example:

the relation between the Carroll geometry (6.55) and Carrollian structures previously constructed in [41] and [42]. The analysis here presented could be useful to find the asymptotic symmetries of general Newton-Cartan and Carroll geometries.

Given the relation between IHM and Drinfeld-Sokolov reduction, it would be useful to do a systematic classification of all possible IHM and to study all possible boundary actions.

An important future direction of this work is also the formulation of the post-Newtonian as well as post-Carrollian corrections of two-dimensional gravity and their boundary theories following recent results that have been obtained in three and four-dimensional gravity $[45,46,67-69]$. This is a work in progress. 
Other possible future directions are the study of the relation of the NR Schwarzian with a novel regime of the SYK model, the quantisation of the NR JT action and its Carrollian counterpart, their relation to matrix models along the lines of [64], and their supersymmetric extensions.

\section{Acknowledgments}

We thank Glenn Barnich, Jaume Gomis, Marc Henneaux, Axel Kleinschmidt, Arash Ranjbar, Mauricio Valenzuela and Jorge Zanelli for enlightening comments and discussions. We specially acknowledge Luis Avilés for taking active part in the initial stages of this work. JG has been supported in part by MINECO FPA2016-76005-C2-1-P and PID2019105614GB-C21 and from the State Agency for Research of the Spanish Ministry of Science and Innovation through the Unit of Excellence Maria de Maeztu 2020-203 award to the Institute of Cosmos Sciences (CEX2019-000918-M). DH is partially funded by ANID grant \#21160649. The Centro de Estudios Científicos (CECs) is funded by the Chilean Government through the Centers of Excellence Base Financing Program of ANID. This work was partially supported by FNRS-Belgium (conventions FRFC PDRT.1025.14 and IISN 4.4503.15), as well as by funds from the Solvay Family.

\section{A Conventions for (A) $\mathrm{dS}_{2}$}

The $(\mathrm{A}) \mathrm{dS}_{2}$ algebra and its invariant bilinear form are defined as

$$
\begin{aligned}
{\left[M_{A B}, M_{C D}\right] } & =g_{A D} M_{B C}-g_{A C} M_{B D}-g_{B D} M_{A C}+g_{B C} M_{A D}, \\
\left\langle M_{A B}, M_{C D}\right\rangle & =\tilde{\gamma}_{0}\left(g_{A D} g_{B C}-g_{A C} g_{B D}\right),
\end{aligned}
$$

where $A=0,1,2$, and $g_{A B}$ is the $(\mathrm{A}) \mathrm{dS}_{2}$ metric

$$
g_{A B}=\operatorname{diag}(-,+,-\sigma)
$$

Here $\sigma=+1$ defines the $\operatorname{AdS}_{2}$ algebra $\mathfrak{s} o(2,1)$, while $\sigma=-1$ corresponds to the $\mathrm{dS}_{2}$ case $\mathfrak{s o}(1,2)$. Usually one redefines the generators in the $(\mathrm{A}) \mathrm{dS}_{2}$ basis as

$$
\tilde{J}_{a b}=M_{a b}, \quad \tilde{P}_{a}=\sqrt{|\tilde{\Lambda}|} M_{2 a}, \quad a=0,1,
$$

where we have introduced the cosmological constant $\tilde{\Lambda}$

$$
\tilde{\Lambda}=-\sigma|\tilde{\Lambda}|
$$

which defines (A)dS radius $\ell=1 / \sqrt{|\tilde{\Lambda}|}$. The definitions (A.1) then take the form

$$
\begin{aligned}
{\left[\tilde{J}_{a b}, \tilde{P}_{c}\right] } & =\eta_{b c} \tilde{P}_{a}-\eta_{a c} \tilde{P}_{b}, & {\left[\tilde{P}_{a}, \tilde{P}_{b}\right] } & =-\tilde{\Lambda} \tilde{J}_{a b}, \\
\left\langle\tilde{J}_{a b}, \tilde{J}_{c d}\right\rangle & =\tilde{\gamma}_{0}\left(\eta_{a d} \eta_{b c}-\eta_{a c} \eta_{b d}\right), & \left\langle\tilde{P}_{a}, \tilde{P}_{b}\right\rangle & =-\tilde{\gamma}_{0} \tilde{\Lambda} \eta_{a b},
\end{aligned}
$$


where $\eta_{a b}=\operatorname{diag}(-,+)$ is the two-dimensional Minkowski metric. We can further simplify these relations by defining the dual generator

$$
\tilde{J}=-\frac{1}{2} \epsilon^{a b} \tilde{J}_{a b} \quad \Longrightarrow \quad \tilde{J}_{a b}=\epsilon_{a b} \tilde{J}, \quad\left(\epsilon_{01}=-\epsilon_{10}=1\right),
$$

which leads to

$$
\begin{aligned}
{\left[\tilde{J}, \tilde{P}_{a}\right] } & =\epsilon_{a}{ }^{b} \tilde{P}_{b} & {\left[\tilde{P}_{a}, \tilde{P}_{b}\right] } & =-\tilde{\Lambda} \epsilon_{a b} \tilde{J}, \\
\langle\tilde{J}, \tilde{J}\rangle & =\tilde{\gamma}_{0}, & \left\langle\tilde{P}_{a}, \tilde{P}_{b}\right\rangle & =-\tilde{\gamma}_{0} \tilde{\Lambda} \eta_{a b} .
\end{aligned}
$$

Both $\mathrm{AdS}_{2}$ and $\mathrm{dS}_{2}$ are isomorphic to the $\mathfrak{s l}(2, \mathbb{R})$ algebra

$$
[\tilde{\mathcal{D}}, \tilde{\mathcal{H}}]=\tilde{\mathcal{H}}, \quad[\tilde{\mathcal{D}}, \tilde{\mathcal{K}}]=-\tilde{\mathcal{K}}, \quad[\tilde{\mathcal{H}}, \tilde{\mathcal{K}}]=2 \tilde{\mathcal{D}}
$$

Setting $\sigma=-1$ in (A.4), we find that in the $\mathrm{AdS}_{2}$ case we can define

$$
\tilde{\mathcal{D}}=\ell \tilde{P}_{1}, \quad \tilde{\mathcal{H}}=\ell \tilde{P}_{0}+\tilde{J}, \quad \tilde{\mathcal{K}}=\ell \tilde{P}_{0}-\tilde{J},
$$

which leads to the $\mathfrak{s l}(2, \mathbb{R})$ commutation relations (A.8). In the $\mathrm{dS}_{2}$ case $(\sigma=1)$, the conformal change of basis that leads to $\mathfrak{s l}(2, \mathbb{R})$ reads

$$
\tilde{\mathcal{D}}=\ell \tilde{P}_{0}, \quad \tilde{\mathcal{H}}=\ell \tilde{P}_{1}+\tilde{J}, \quad \tilde{\mathcal{K}}=\ell \tilde{P}_{1}-\tilde{J} .
$$

In both cases the $\mathfrak{s l}(2, \mathbb{R})$ pairing follows from $($ A.7) and is given by

$$
\langle\tilde{\mathcal{D}}, \tilde{\mathcal{D}}\rangle=\tilde{\gamma}_{0}, \quad\langle\tilde{\mathcal{H}}, \tilde{\mathcal{K}}\rangle=-2 \tilde{\gamma}_{0} .
$$

One can consider the following matrix representation

$$
\tilde{\mathcal{D}}=\frac{1}{2}\left(\begin{array}{rr}
1 & 0 \\
0 & -1
\end{array}\right), \quad \tilde{\mathcal{K}}=\left(\begin{array}{cc}
0 & -1 \\
0 & 0
\end{array}\right), \quad \tilde{\mathcal{H}}=\left(\begin{array}{ll}
0 & 0 \\
1 & 0
\end{array}\right),
$$

for which the bilinear form can be expressed simply as $\langle\cdot, \cdot\rangle=2 \tilde{\gamma}_{0} \operatorname{Tr}(\cdot)$.

\section{B Building blocks for the NR second order formulation}

In this appendix, we compute the NR second-order of JT gravity. The starting point is to consider an $\varepsilon$-expansion for the metric $g_{\mu \nu}[39,43-45,51-54]$ and then from the metric compatibility, we find the Levi-Civita connections at the order in $\varepsilon$ which we are interested.

\section{B.1 NR affine connections}

Let us start with the expansion (3.1) of the metric and its inverse

$$
g_{\mu \nu}=\frac{1}{\varepsilon^{2}} \stackrel{(2)}{g}_{\mu \nu}+\stackrel{(0)}{g}_{\mu \nu}, \quad \quad g^{\mu \nu}=\stackrel{(0)}{g}^{\mu \nu}+\varepsilon^{2} \stackrel{(-2)}{g}_{\mu \nu} .
$$

From the relation $g_{\nu \rho} g^{\rho \mu}=\delta_{\nu}^{\mu}$, we get order-by-order the identities

$$
\stackrel{(2)}{g}_{\mu \rho} \stackrel{(0)}{g}^{\rho \nu}=0, \quad \stackrel{(0)}{g}_{\nu \rho} \stackrel{(0)}{g} \rho \mu+\stackrel{(2)}{g}_{\nu \rho} \stackrel{(-2)}{g} \rho \mu=\delta_{\nu}^{\mu}, \quad \stackrel{(0)}{g}_{\mu \rho} \stackrel{(-2)}{g} \rho \nu=0 .
$$


Now we demand the metric compatibility condition for the Lorentzian metric, namely

$$
\nabla_{\rho} g_{\mu \nu}=0
$$

The condition (B.3) implies the following set of equations

$$
\begin{array}{rl}
\stackrel{(0)}{\nabla}_{\rho} \stackrel{(0)}{g}_{\mu \nu}-2 \stackrel{(-2)}{\Gamma}_{\rho}^{\lambda} \stackrel{(2)}{g}_{\mu) \lambda} & =0, \\
\stackrel{(0)}{\nabla}_{\rho}^{(2)} \stackrel{(2)}{g}_{\mu \nu}-2 & 2 \stackrel{(2)}{\Gamma}_{\rho(\nu}^{\lambda} \stackrel{(0)}{g}_{\mu) \lambda}=0, \\
2 \stackrel{(2)}{\Gamma}_{\rho}^{\lambda} \stackrel{(2)}{(\nu)}_{\mu) \lambda}=0, \\
2 \stackrel{(-2)}{\Gamma}_{\rho}^{\lambda} \stackrel{(0)}{g}_{\mu) \lambda}=0 .
\end{array}
$$

In order to solve the affine connections, we proceed in the standard way by permuting indexes in (B.4), we find

$$
\begin{aligned}
\frac{1}{2}\left(\partial_{\mu} \stackrel{(2)}{g}_{\nu \rho}+\partial_{\nu} \stackrel{(2)}{g}_{\mu \rho}-\partial_{\rho} \stackrel{(2)}{g}_{\mu \nu}\right) & =\stackrel{(2)}{g}_{\rho \lambda} \stackrel{(0)}{\Gamma}_{\mu \nu}^{\lambda}+\stackrel{(0)}{g}_{\rho \lambda} \stackrel{(2)}{\Gamma}_{\mu \nu}, \\
\frac{1}{2}\left(\partial_{\mu} \stackrel{(0)}{g}_{\nu \rho}+\partial_{\nu} \stackrel{(0)}{g}_{\mu \rho}-\partial_{\rho} \stackrel{(0)}{g}_{\mu \nu}\right) & =\stackrel{(0)}{g}_{\rho \lambda} \stackrel{(0)}{\Gamma}_{\mu \nu}^{\lambda}+\stackrel{(2)}{g}_{\rho \lambda} \stackrel{(-2)}{\Gamma}{ }_{\mu \nu}^{\lambda}, \\
\stackrel{(2)}{\Gamma}_{\mu \nu}^{\lambda} \stackrel{(2)}{g}_{\rho \lambda} & =0 \\
\stackrel{(-2)}{\Gamma}_{\mu \nu} \stackrel{(0)}{g}_{\rho \lambda} & =0 .
\end{aligned}
$$

Using the relations (B.2) in the last equations, we get the following expressions for the terms in the expansion Levi-Civita affine connection

$$
\begin{aligned}
& \stackrel{(0)}{\Gamma}_{\mu \nu}^{\alpha}=\frac{1}{2} \stackrel{(0)}{g} \rho \alpha\left(\partial_{\mu} \stackrel{(0)}{g}_{\nu \rho}+\partial_{\nu} \stackrel{(0)}{g}_{\mu \rho}-\partial_{\rho} \stackrel{(0)}{g}_{\mu \nu}\right) \\
& +\frac{1}{2} \stackrel{(-2)}{g} \rho \alpha\left(\partial_{\mu} \stackrel{(2)}{g}_{\nu \rho}+\partial_{\nu} \stackrel{(2)}{g}_{\mu \rho}-\partial_{\rho} \stackrel{(2)}{g}_{\mu \nu}\right), \\
& \stackrel{(-2)}{\Gamma}_{\mu \nu}^{\alpha}=\frac{1}{2} \stackrel{(-2)}{g} \rho \alpha\left(\partial_{\mu} \stackrel{(0)}{g}_{\nu \rho}+\partial_{\nu} \stackrel{(0)}{g}_{\mu \rho}-\partial_{\rho} \stackrel{(0)}{g}_{\mu \nu}\right) \text {, } \\
& \stackrel{(2)}{\Gamma}_{\mu \nu}^{\alpha}=\frac{1}{2} \stackrel{(0)}{g} \rho \alpha\left(\partial_{\mu} \stackrel{(2)}{g}_{\nu \rho}+\partial_{\nu} \stackrel{(2)}{g}_{\mu \rho}-\partial_{\rho} \stackrel{(2)}{g}_{\mu \nu}\right) \text {. }
\end{aligned}
$$

\section{B.2 Ricci scalar}

Now we compute the different orders of the Ricci scalar expansion appearing in (3.14). In contrast to higher dimensions, we show that in two space-time dimensions the divergent terms vanish identically simply using the completeness relations of the fields of NewtonCartan geometry.

Starting from the standard relativistic formula of the Ricci scalar

$$
\mathcal{R}=g^{\mu \nu}\left(\partial_{\lambda} \Gamma_{\mu \nu}^{\lambda}-\partial_{\nu} \Gamma_{\lambda \mu}^{\lambda}+\Gamma_{\lambda \alpha}^{\lambda} \Gamma_{\mu \nu}^{\alpha}-\Gamma_{\nu \alpha}^{\lambda} \Gamma_{\lambda \mu}^{\alpha}\right)
$$


and substituting the expansions (B.1) and (B.9), we find the following expansion for the Ricci scalar

$$
\mathcal{R}=\frac{1}{\varepsilon^{4}} \stackrel{(4)}{\mathcal{R}}+\frac{1}{\varepsilon^{2}} \stackrel{(2)}{\mathcal{R}}+\stackrel{(0)}{\mathcal{R}}+\varepsilon^{2} \stackrel{(-2)}{\mathcal{R}}+\mathcal{O}\left(\varepsilon^{4}\right)
$$

where

$$
\begin{aligned}
& \stackrel{(4)}{\mathcal{R}}=2 \stackrel{(0)}{g} \mu \nu \stackrel{(2)}{\Gamma} \underset{\alpha \lambda}{\lambda} \stackrel{(2)}{\Gamma}{ }_{\nu \mu}^{\alpha}, \\
& \stackrel{(2)}{\mathcal{R}}=2 \stackrel{(-2)}{g} \mu \nu\left(\stackrel{(2)}{\Gamma}_{\alpha \lambda}^{\lambda} \stackrel{(2)}{\Gamma}_{\nu \mu}^{\alpha}\right)+2 \stackrel{(0)}{g} \mu \nu\left(\partial_{\lambda} \stackrel{(2)}{\Gamma}_{\nu \mu}^{\lambda}+\stackrel{(0)}{\Gamma}_{\alpha \lambda}^{\lambda} \stackrel{(2)}{\Gamma}_{\nu \mu}^{\alpha}+\stackrel{(2)}{\Gamma}_{\alpha \lambda}^{\lambda} \stackrel{(0)}{\Gamma}_{\nu \mu}^{\alpha}\right) \\
& \stackrel{(0)}{\mathcal{R}}=2 \stackrel{(-2)}{g} \mu \nu\left(\partial_{[\lambda} \stackrel{(2)}{\Gamma}_{\nu] \mu}^{\lambda}+\stackrel{(0)}{\Gamma}_{\alpha[\lambda}^{\lambda} \stackrel{(2)}{\Gamma}_{\nu] \mu}^{\alpha}+\stackrel{(2)}{\Gamma}_{\alpha[\lambda}^{\lambda} \stackrel{(0)}{\Gamma}_{\nu] \mu}^{\alpha}\right) \\
& +2 \stackrel{(0)}{g} \mu \nu\left(\partial_{[\lambda} \stackrel{(0)}{\Gamma} \stackrel{\lambda}{\nu] \mu}_{\nu}+\stackrel{(0)}{\Gamma} \underset{\alpha[\lambda}{\lambda} \stackrel{(0)}{\Gamma}_{\nu] \mu}^{\alpha}+\stackrel{(-2)}{\Gamma} \underset{\alpha[\lambda}{\lambda} \stackrel{(2)}{\Gamma}{ }_{\nu] \mu}^{\alpha}+\stackrel{(2)}{\Gamma}_{\alpha[\lambda}^{\lambda} \stackrel{(-2)}{\Gamma}_{\nu] \mu}^{\alpha}\right), \\
& \stackrel{(-2)}{\mathcal{R}}=2 \stackrel{(-2)}{g} \mu \nu \quad\left(\partial_{[\lambda} \stackrel{(0)}{\Gamma} \underset{\nu] \mu}{\lambda}+\stackrel{(0)}{\Gamma}_{\alpha[\lambda}^{\lambda} \stackrel{(0)}{\Gamma}_{\nu] \mu}^{\alpha}+\stackrel{(-2)}{\Gamma}_{\alpha[\lambda}^{\lambda} \stackrel{(2)}{\Gamma}_{\nu] \mu}^{\alpha}+\stackrel{(2)}{\Gamma}_{\alpha[\lambda}^{\lambda} \stackrel{(-2)}{\Gamma}_{\nu] \mu}^{\alpha}\right) \\
& +2 \stackrel{(0)}{g} \mu \nu\left(\partial_{[\lambda} \stackrel{(-2)}{\Gamma} \underset{\nu] \mu}{\lambda}+\stackrel{(0)}{\Gamma} \underset{\alpha[\lambda}{\lambda} \stackrel{(-2)}{\Gamma} \underset{\nu] \mu}{\alpha}+\stackrel{(-2)}{\Gamma}_{\alpha[\lambda}^{\lambda} \stackrel{(0)}{\Gamma} \underset{\nu] \mu}{\alpha}\right) .
\end{aligned}
$$

Using the result $\stackrel{(2)}{\Gamma}{ }_{\mu \nu}^{\lambda}=0$ found in (3.19), the divergent terms vanish identically. The first term in the expansion in thus the finite term

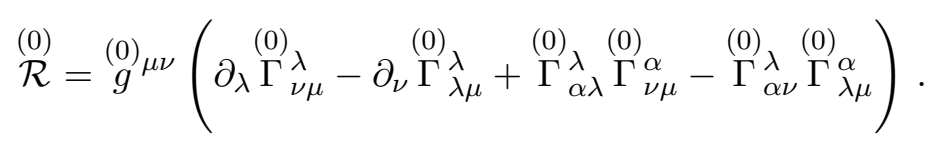

Note that using (3.19), the terms with derivatives in (B.16) take the form

$$
\begin{aligned}
\partial_{\lambda} \stackrel{(0)}{\Gamma}{ }_{\mu \nu}^{\lambda}-\partial_{\nu} \stackrel{(0)}{\Gamma}{ }_{\mu \lambda}^{\lambda}= & \partial_{\lambda} \tau^{\lambda} \partial_{\mu} \tau_{\nu}+\partial_{\lambda} e^{\lambda}\left(\partial_{\mu} e_{\nu}+\omega_{\mu} \tau_{\nu}\right)+e^{\lambda}\left(\partial_{\lambda} \omega_{\mu} \tau_{\nu}+\omega_{\mu} \partial_{\lambda} \tau_{\nu}\right) \\
& -\partial_{\nu} \tau^{\lambda} \partial_{\lambda} \tau_{\mu}-\partial_{\nu} e^{\lambda}\left(\partial_{\lambda} e_{\mu}+\omega_{\lambda} \tau_{\mu}\right)+e^{\lambda}\left(\partial_{\nu} \omega_{\lambda} \tau_{\mu}+\omega_{\lambda} \partial_{\nu} \tau_{\mu}\right)
\end{aligned}
$$

Now, from the vielbein postulate (3.15) together with the expressions (3.7) and (3.16) we get the following set of equations

$$
\begin{aligned}
\partial_{\mu} \tau_{\nu}-\stackrel{(0)}{\Gamma}{ }_{\mu \nu}^{\lambda} \tau_{\lambda}=0, & \text { (B.19a) } & \partial_{\mu} \tau^{\nu}-\omega_{\mu} e^{\nu}+\stackrel{(0)}{\Gamma}_{\mu \lambda}^{\nu} \tau^{\lambda}=0 \\
\partial_{\mu} e_{\nu}+\omega_{\mu} \tau_{\nu}-\stackrel{(0)}{\Gamma}{ }_{\mu \nu}^{\lambda} e_{\lambda}=0, & \text { (B.19b) } & \partial_{\mu} e^{\nu}+\stackrel{(0)}{\Gamma}{ }_{\mu \lambda}^{\nu} e^{\lambda}=0
\end{aligned}
$$

Using (B.19b) and (B.19c), we can rewrite some terms in (B.17) as follows

$$
\begin{aligned}
& \partial_{\lambda} \tau^{\lambda} \partial_{\mu} \tau_{\nu}+\partial_{\lambda} e^{\lambda}\left(\partial_{\mu} e_{\nu}+\omega_{\mu} \tau_{\nu}\right)=\omega_{\lambda} e^{\lambda} \stackrel{(0)}{\Gamma_{\mu}^{\sigma}} \tau_{\mu \nu}-\stackrel{(0)}{\Gamma}{ }_{\lambda \rho}^{\lambda} \stackrel{(0)}{\Gamma}_{\mu \nu}^{\rho}, \\
& \partial_{\nu} \tau^{\lambda} \partial_{\lambda} \tau_{\mu}+\partial_{\nu} e^{\lambda}\left(\partial_{\lambda} e_{\mu}+\omega_{\lambda} \tau_{\mu}\right)=\omega_{\nu} e^{\lambda} \stackrel{(0)}{\Gamma_{\lambda \mu}^{\sigma}} \tau_{\sigma}-\stackrel{(0)}{\Gamma}{ }_{\nu \rho} \stackrel{(0)}{\Gamma}_{\lambda \mu}^{\rho}
\end{aligned}
$$


This last fact is quite remarkable because helps us to cancel out the quadratic terms of the affine connection $\stackrel{(0)}{\Gamma}_{\mu \nu}^{\lambda}$ appearing in (B.16). Then, plugging (B.20) and (B.21) into (B.16), we find

$$
\stackrel{(0)}{\mathcal{R}}=0 .
$$

Finally, we look at the last relevant term in the Ricci scalar expansion (B.11)

$$
\begin{aligned}
\stackrel{(-2)}{\mathcal{R}}= & 2^{(-2)} g \nu \nu\left(\partial_{[\lambda} \stackrel{(0)}{\Gamma} \underset{\nu] \mu}{\lambda}+\stackrel{(0)}{\Gamma} \underset{\rho[\lambda}{\lambda} \stackrel{(0)}{\Gamma} \underset{\nu] \mu}{\rho}\right) \\
& +2 \stackrel{(0)}{g} \mu \nu\left(\partial_{[\lambda} \stackrel{(-2)}{\Gamma} \underset{\nu] \mu}{\lambda}+\stackrel{(0)}{\Gamma} \underset{\rho[\lambda}{\lambda} \stackrel{(-2)}{\Gamma} \underset{\nu] \mu}{\rho}+\stackrel{(-2)}{\Gamma} \underset{\rho[\lambda}{\lambda} \stackrel{(0)}{\Gamma}{ }_{\nu] \mu}^{\rho}\right) .
\end{aligned}
$$

The terms involving $\stackrel{(0)}{\Gamma}{ }_{\mu \nu}^{\lambda}$ are computed in the same way as done before. This yields

$$
2^{(-2)} \mu \nu\left(\partial_{[\lambda} \stackrel{(0)}{\Gamma} \underset{\nu] \mu}{\lambda}+\stackrel{(0)}{\Gamma} \underset{\rho[\lambda}{\lambda} \stackrel{(0)}{\Gamma} \underset{\nu] \mu}{\rho}\right)=2 \tau^{\mu} e^{\nu} \partial_{[\mu} \omega_{\nu]} .
$$

We now need to compute the terms proportional to $\stackrel{(-2)}{\Gamma} \underset{\mu \nu}{\lambda}$. In order to do so, let us consider the following formulae obtained from the user (3.9) and (3.16) in the relativistic inverse vielbein postulate

$$
\begin{array}{r}
\partial_{\mu} \tau^{\nu}-\omega_{\mu} e^{\nu}+\stackrel{(0)}{\Gamma}_{\mu \rho}^{\nu} \tau^{\rho}=0, \\
\partial_{\mu} e^{\nu}+\stackrel{(0)}{\Gamma}_{\mu \rho}^{\nu} e^{\rho}=0, \\
\omega_{\mu} \tau^{\nu}-\stackrel{(0)}{\Gamma}_{\mu \rho}^{\nu} e^{\rho}=0 \\
\partial_{\mu} m_{\nu}+\omega_{\mu} e_{\nu}-\stackrel{(0)}{\Gamma}_{\mu \nu}^{\rho} m_{\rho}-\stackrel{(-2)}{\Gamma}{ }_{\mu \nu} \tau_{\rho}=0 \\
\omega_{\mu} m_{\nu}-\stackrel{(-2)}{\Gamma} \underset{\mu \nu}{\rho} e_{\rho}=0 .
\end{array}
$$

We start computing the term,

$$
\begin{aligned}
\partial_{\lambda} \stackrel{(-2)}{\Gamma} \underset{\mu \nu}{\lambda}= & \partial_{\lambda} \tau^{\lambda}\left(\partial_{\mu} m_{\nu}+\omega_{\mu} e_{\nu}-\stackrel{(0)}{\Gamma}_{\mu \nu}^{\sigma} m_{\sigma}\right) \\
& +\tau^{\lambda}\left(\partial_{\lambda} \partial_{\mu} m_{\nu}+\partial_{\lambda} \omega_{\mu} e_{\nu}+\omega_{\mu} \partial_{\lambda} e_{\nu}-\partial_{\lambda} \stackrel{(0)}{\Gamma}_{\mu \nu}^{\sigma} m_{\sigma}-\stackrel{(0)}{\Gamma}_{\mu \nu}^{\sigma} \partial_{\lambda} m_{\sigma}\right) \\
& +\partial_{\lambda} e^{\lambda} \omega_{\mu} m_{\nu}+e^{\lambda}\left(\partial_{\lambda} \omega_{\mu} m_{\nu}+\omega_{\mu} \partial_{\lambda} m_{\nu}\right) .
\end{aligned}
$$

Using (B.25a) and (B.25d), we can rewrite the first term on the right hand side of (B.26) as

$$
\begin{aligned}
\partial_{\lambda} \tau^{\lambda}\left(\partial_{\mu} m_{\nu}+\omega_{\mu} e_{\nu}-\stackrel{(0)}{\Gamma}{ }_{\mu \nu}^{\sigma} m_{\sigma}\right) & =\left(\omega_{\lambda} e^{\lambda}-\stackrel{(0)}{\Gamma} \underset{\lambda \rho}{\lambda} \tau^{\rho}\right) \stackrel{(-2)}{\Gamma} \underset{\mu \nu}{\sigma} \tau_{\sigma}, \\
& =\omega_{\lambda} e^{\lambda} \stackrel{(-2)}{\Gamma} \underset{\mu \nu}{\sigma} \tau_{\sigma}-\stackrel{(0)}{\Gamma}{ }_{\lambda \rho}^{\lambda} \stackrel{(-2)}{\Gamma} \underset{\mu \nu}{\rho}+\stackrel{(0)}{\Gamma}{ }_{\lambda \rho}^{\lambda} \stackrel{(-2)}{\Gamma}{ }_{\mu \nu} e^{\rho} e_{\sigma},
\end{aligned}
$$


where we have used the completeness relation (3.10). Using (B.25d), we can rewrite the last term in the second line of (B.26), namely

$$
\begin{aligned}
\tau^{\lambda} \stackrel{(0)}{\Gamma}_{\mu \nu}^{\sigma} \partial_{\lambda} m_{\sigma} & =\tau^{\lambda} \stackrel{(0)}{\Gamma}_{\mu \nu}^{\sigma}\left(\stackrel{(0)}{\Gamma}{ }_{\lambda \sigma}^{\rho} m_{\rho}+\stackrel{(-2)}{\Gamma} \underset{\lambda \sigma}{\rho} \tau_{\rho}-\omega_{\lambda} e_{\sigma}\right) \\
& =\tau^{\lambda} \stackrel{(0)}{\Gamma}_{\mu \nu}^{\sigma} \stackrel{(0)}{\Gamma}{ }_{\lambda \sigma}^{\rho} m_{\rho}+\stackrel{(0)}{\Gamma}{ }_{\mu \nu}^{\rho} \stackrel{(-2)}{\Gamma}{ }_{\lambda \rho}^{\lambda}-\stackrel{(0)}{\Gamma}_{\mu \nu}^{\sigma} \stackrel{(-2)}{\Gamma}_{\lambda \sigma}^{\rho} e^{\lambda} e_{\rho}-\stackrel{(0)}{\Gamma}_{\mu \nu}^{\sigma} \tau^{\lambda} \omega_{\lambda} e_{\sigma}
\end{aligned}
$$

Plugging (B.27) and (B.28) into (B.26), we get

$$
\begin{aligned}
& \partial_{\lambda} \stackrel{(-2)}{\Gamma} \underset{\mu \nu}{\lambda}=\omega_{\lambda} e^{\lambda} \stackrel{(-2)}{\Gamma} \underset{\mu \nu}{\sigma} \tau_{\sigma}-\stackrel{(0)}{\Gamma} \underset{\lambda \rho}{\lambda} \stackrel{(-2)}{\Gamma} \stackrel{\rho}{\mu \nu}_{\mu \nu}+\stackrel{(0)}{\Gamma}{ }_{\lambda \rho}^{\lambda} \stackrel{(-2)}{\Gamma} \underset{\mu \nu}{\sigma} e^{\rho} e_{\sigma} \\
& +\tau^{\lambda}\left(\partial_{\lambda} \partial_{\mu} m_{\nu}+\partial_{\lambda} \omega_{\mu} e_{\nu}+\omega_{\mu} \partial_{\lambda} e_{\nu}-\partial_{\lambda} \stackrel{(0)}{\Gamma}_{\mu \nu}^{\sigma} m_{\sigma}\right) \\
& -\tau^{\lambda} \stackrel{(0)}{\Gamma}_{\mu \nu}^{\sigma} \stackrel{(0)}{\Gamma}_{\lambda \sigma}^{\rho} m_{\rho}-\stackrel{(0)}{\Gamma}_{\mu \nu}^{\rho} \stackrel{(-2)}{\Gamma}{ }_{\lambda \rho}^{\lambda}+\stackrel{(0)}{\Gamma}{ }_{\mu \nu}^{\sigma} \stackrel{(-2)}{\Gamma} \underset{\lambda \sigma}{\rho} e^{\lambda} e_{\rho}+\stackrel{(0)}{\Gamma}{ }_{\mu \nu}^{\sigma} \tau^{\lambda} \omega_{\lambda} e_{\sigma} \\
& +\partial_{\lambda} e^{\lambda} \omega_{\mu} m_{\nu}+e^{\lambda}\left(\partial_{\lambda} \omega_{\mu} m_{\nu}+\omega_{\mu} \partial_{\lambda} m_{\nu}\right) \text {. }
\end{aligned}
$$

Replacing these results in (B.23) a little algebra leads to

$$
\begin{aligned}
\stackrel{(-2)}{\mathcal{R}}= & 4 \tau^{\mu} e^{\nu} \partial_{[\mu} \omega_{\nu]} \\
& +h^{\mu \nu}\left(e^{\rho} e_{\sigma}\left[\stackrel{(0)}{\Gamma}{ }_{\lambda \rho}^{\lambda} \stackrel{(0)}{\Gamma}_{\mu \nu}^{\sigma}-\stackrel{(0)}{\Gamma}_{\nu \rho}^{\lambda} \stackrel{(0)}{\Gamma}_{\lambda \mu}^{\sigma}\right]+\partial_{\lambda} e^{\lambda} \omega_{\mu} m_{\nu}-\partial_{\nu} e^{\lambda} \omega_{\lambda} m_{\mu}\right)
\end{aligned}
$$

Finally, using the identities (B.25), we find

$$
\stackrel{(-2)}{\mathcal{R}}=4 \tau^{\mu} e^{\nu} \partial_{[\mu} \omega_{\nu]}
$$

Open Access. This article is distributed under the terms of the Creative Commons Attribution License (CC-BY 4.0), which permits any use, distribution and reproduction in any medium, provided the original author(s) and source are credited.

\section{References}

[1] S. Sachdev and J. Ye, Gapless spin fluid ground state in a random, quantum Heisenberg magnet, Phys. Rev. Lett. 70 (1993) 3339 [cond-mat/9212030] [INSPIRE].

[2] A. Kitaev, A simple model of quantum holography (part 1), talk at KITP, April 7, 2015, http://online.kitp.ucsb.edu/online/entangled15/kitaev/.

[3] A. Kitaev, A simple model of quantum holography (part 2), talk at KITP, May 27, 2015, http://online.kitp.ucsb.edu/online/entangled15/kitaev2/.

[4] A. Kitaev and S.J. Suh, The soft mode in the Sachdev-Ye-Kitaev model and its gravity dual, JHEP 05 (2018) 183 [arXiv: 1711.08467] [INSPIRE].

[5] C. Teitelboim, Gravitation and Hamiltonian Structure in Two Space-Time Dimensions, Phys. Lett. B 126 (1983) 41 [INSPIRE]. 
[6] R. Jackiw, Lower Dimensional Gravity, Nucl. Phys. B 252 (1985) 343 [InSPIRE].

[7] J. Maldacena and D. Stanford, Remarks on the Sachdev-Ye-Kitaev model, Phys. Rev. D 94 (2016) 106002 [arXiv:1604.07818] [INSPIRE].

[8] D. Stanford and E. Witten, Fermionic Localization of the Schwarzian Theory, JHEP 10 (2017) 008 [arXiv: 1703.04612] [INSPIRE].

[9] J. Maldacena, D. Stanford and Z. Yang, Conformal symmetry and its breaking in two dimensional Nearly Anti-de-Sitter space, PTEP 2016 (2016) 12C104 [arXiv:1606.01857] [INSPIRE].

[10] J. Engelsöy, T.G. Mertens and H. Verlinde, An investigation of $A d S_{2}$ backreaction and holography, JHEP 07 (2016) 139 [arXiv:1606.03438] [INSPIRE].

[11] K. Jensen, Chaos in AdS 2 Holography, Phys. Rev. Lett. 117 (2016) 111601 [arXiv: 1605.06098] [INSPIRE].

[12] S. Sachdev, Bekenstein-Hawking Entropy and Strange Metals, Phys. Rev. X 5 (2015) 041025 [arXiv: 1506.05111] [INSPIRE].

[13] R.A. Davison, W. Fu, A. Georges, Y. Gu, K. Jensen and S. Sachdev, Thermoelectric transport in disordered metals without quasiparticles: The Sachdev-Ye-Kitaev models and holography, Phys. Rev. B 95 (2017) 155131 [arXiv:1612.00849] [InSPIRE].

[14] K. Bulycheva, A note on the SYK model with complex fermions, JHEP 12 (2017) 069 [arXiv: 1706.07411] [INSPIRE].

[15] P. Chaturvedi, Y. Gu, W. Song and B. Yu, A note on the complex SYK model and warped CFTs, JHEP 12 (2018) 101 [arXiv: 1808.08062] [INSPIRE].

[16] Y. Gu, A. Kitaev, S. Sachdev and G. Tarnopolsky, Notes on the complex Sachdev-Ye-Kitaev model, JHEP 02 (2020) 157 [arXiv: 1910.14099] [INSPIRE].

[17] V. Godet and C. Marteau, New boundary conditions for AdS 2 , JHEP 12 (2020) 020 [arXiv: 2005.08999] [INSPIRE].

[18] H. Afshar, H.A. González, D. Grumiller and D. Vassilevich, Flat space holography and the complex Sachdev-Ye-Kitaev model, Phys. Rev. D 101 (2020) 086024 [arXiv:1911.05739] [INSPIRE].

[19] A.R. Brown, H. Gharibyan, H.W. Lin, L. Susskind, L. Thorlacius and Y. Zhao, Complexity of Jackiw-Teitelboim gravity, Phys. Rev. D 99 (2019) 046016 [arXiv:1810.08741] [INSPIRE].

[20] L. Susskind, Complexity and Newton's Laws, Front. in Phys. 8 (2020) 262 [arXiv: 1904.12819] [INSPIRE].

[21] J.L.F. Barbón, J. Martin-Garcia and M. Sasieta, Proof of a Momentum/Complexity Correspondence, Phys. Rev. D 102 (2020) 101901 [arXiv:2006.06607] [INSPIRE].

[22] D.E. Berenstein, J.M. Maldacena and H.S. Nastase, Strings in flat space and pp waves from $N=4$ superYang-Mills, JHEP 04 (2002) 013 [hep-th/0202021] [INSPIRE].

[23] J. Gomis, J. Gomis and K. Kamimura, Non-relativistic superstrings: A New soluble sector of $A d S_{5} \times S^{5}, J H E P 12(2005) 024$ [hep-th/0507036] [INSPIRE].

[24] T. Fukuyama and K. Kamimura, Gauge Theory of Two-dimensional Gravity, Phys. Lett. B 160 (1985) 259 [INSPIRE]. 
[25] K. Isler and C.A. Trugenberger, A Gauge Theory of Two-dimensional Quantum Gravity, Phys. Rev. Lett. 63 (1989) 834 [INSPIRE].

[26] A.H. Chamseddine and D. Wyler, Gauge Theory of Topological Gravity in (1+1)-Dimensions, Phys. Lett. B 228 (1989) 75 [InSPIRE].

[27] A. Galajinsky, Schwarzian mechanics via nonlinear realizations, Phys. Lett. B 795 (2019) 277 [arXiv: 1905.01935] [inSPIRE].

[28] S.R. Coleman, J. Wess and B. Zumino, Structure of phenomenological Lagrangians. 1, Phys. Rev. 177 (1969) 2239 [inSPIRE].

[29] C.G. Callan Jr., S.R. Coleman, J. Wess and B. Zumino, Structure of phenomenological Lagrangians. 2, Phys. Rev. 177 (1969) 2247 [INSPIRE].

[30] E.A. Ivanov and V.I. Ogievetsky, The Inverse Higgs Phenomenon in Nonlinear Realizations, Teor. Mat. Fiz. 25 (1975) 164 [InSPIRE].

[31] H. Bacry and J. Levy-Leblond, Possible kinematics, J. Math. Phys. 9 (1968) 1605 [InSPIRE].

[32] J. Derome and J.G. Dubouis, Hooke's symmetries and nonrelativistic cosmological model kinematics, Nuovo Cim. B 9 (1972) 351.

[33] J.M. Lévy-Leblond, Une nouvelle limite non-relativiste du group de Poincaré, Ann. Inst. H. Poincaré 3 (1965) 1.

[34] M. Ba nados, Global charges in Chern-Simons field theory and the (2+1) black hole, Phys. Rev. D 52 (1996) 5816 [hep-th/9405171] [INSPIRE].

[35] E. Cartan, Sur les variétés à connexion affine et la théorie de la relativité généralisée. (première partie), Annales Sci. Ecole Norm. Sup. 40 (1923) 325.

[36] E. Cartan, Sur les variétés à connexion affine et la théorie de la relativité généralisée. (première partie) (Suite), Annales Sci. Ecole Norm. Sup. 41 (1924) 1.

[37] A. Trautman, Sur la Théorie Newtonienne de la Gravitation, Compt. Rend. Acad. Sci. 257 (1963) 617.

[38] P. HAVAS, Four-Dimensional Formulations of Newtonian Mechanics and Their Relation to the Special and the General Theory of Relativity, Rev. Mod. Phys. 36 (1964) 938 [InSPIRE].

[39] H.P. Kuenzle, Galilei and lorentz structures on space-time - comparison of the corresponding geometry and physics, Ann. Inst. H. Poincare Phys. Theor. 17 (1972) 337 [InSPIRE].

[40] K. Kuchar, Gravitation, geometry, and nonrelativistic quantum theory, Phys. Rev. D 22 (1980) 1285 [INSPIRE].

[41] M. Henneaux, Geometry of Zero Signature Space-times, Bull. Soc. Math. Belg. 31 (1979) 47 [INSPIRE].

[42] C. Duval, G.W. Gibbons, P.A. Horvathy and P.M. Zhang, Carroll versus Newton and Galilei: two dual non-Einsteinian concepts of time, Class. Quant. Grav. 31 (2014) 085016 [arXiv: 1402.0657] [INSPIRE].

[43] D. Hansen, J. Hartong and N.A. Obers, Non-Relativistic Gravity and its Coupling to Matter, JHEP 06 (2020) 145 [arXiv:2001.10277] [INSPIRE].

[44] R. De Pietri, L. Lusanna and M. Pauri, Standard and generalized Newtonian gravities as 'gauge' theories of the extended Galilei group. I. The standard theory, Class. Quant. Grav. 12 (1995) 219 [gr-qc/9405046] [INSPIRE]. 
[45] D. Van den Bleeken, Torsional Newton-Cartan gravity from the large $c$ expansion of general relativity, Class. Quant. Grav. 34 (2017) 185004 [arXiv:1703.03459] [INSPIRE].

[46] E. Bergshoeff, J.M. Izquierdo, T. Ortín and L. Romano, Lie Algebra Expansions and Actions for Non-Relativistic Gravity, JHEP 08 (2019) 048 [arXiv: 1904.08304] [INSPIRE].

[47] D. Grumiller, J. Hartong, S. Prohazka and J. Salzer, Limits of JT gravity, JHEP 02 (2021) 134 [arXiv: 2011.13870] [INSPIRE].

[48] A. Barducci, R. Casalbuoni and J. Gomis, Confined dynamical systems with Carroll and Galilei symmetries, Phys. Rev. D 98 (2018) 085018 [arXiv:1804.10495] [InSPIRE].

[49] E. Bergshoeff, J.M. Izquierdo and L. Romano, Carroll versus Galilei from a Brane Perspective, JHEP 10 (2020) 066 [arXiv: 2003.03062] [INSPIRE].

[50] R. Jackiw, Gauge theories for lineal gravities, in International Conference on Interface Between Physics and Mathematics, (1993) [hep-th/9309082] [INSPIRE].

[51] S. Weinberg, Gravitation and Cosmology: Principles and Applications of the General Theory of Relativity, John Wiley and Sons, New York U.S.A. (1972).

[52] G. Dautcourt, Die newtonske gravitationstheorie als strenger grenzfall der allgemeinen relativitätheorie, Acta Phys. Pol 25 (1964) 637.

[53] J. Ehlers, Republication of: On the Newtonian limit of Einstein's theory of gravitation, Gen. Rel. Grav. 51 (2019) 163 [INSPIRE].

[54] G. Dautcourt, On the ultrarelativistic limit of general relativity, Acta Phys. Polon. B 29 (1998) 1047 [gr-qc/9801093] [INSPIRE].

[55] E. Bergshoeff, J. Rosseel and T. Zojer, Newton-Cartan (super)gravity as a non-relativistic limit, Class. Quant. Grav. 32 (2015) 205003 [arXiv:1505.02095] [INSPIRE].

[56] R. Andringa, E. Bergshoeff, S. Panda and M. de Roo, Newtonian Gravity and the Bargmann Algebra, Class. Quant. Grav. 28 (2011) 105011 [arXiv:1011.1145] [INSPIRE].

[57] T. Regge and C. Teitelboim, Role of Surface Integrals in the Hamiltonian Formulation of General Relativity, Annals Phys. 88 (1974) 286 [INSPIRE].

[58] E. Frodden and D. Hidalgo, Surface Charges Toolkit for Gravity, Int. J. Mod. Phys. D 29 (2020) 2050040 [arXiv: 1911.07264] [InSPIRE].

[59] D. Grumiller, M. Leston and D. Vassilevich, Anti-de Sitter holography for gravity and higher spin theories in two dimensions, Phys. Rev. D 89 (2014) 044001 [arXiv:1311.7413] [INSPIRE].

[60] W. Siegel, Conformal Invariance of Extended Spinning Particle Mechanics, Int. J. Mod. Phys. A 3 (1988) 2713 [inSPIRE].

[61] J. Gomis, J. Herrero, K. Kamimura and J. Roca, Diffeomorphisms, nonlinear W symmetries and particle models, Annals Phys. 244 (1995) 67 [hep-th/9309048] [INSPIRE].

[62] A.M. Polyakov, Gauge Transformations and Diffeomorphisms, Int. J. Mod. Phys. A 5 (1990) 833 [INSPIRE].

[63] V.G. Drinfeld and V.V. Sokolov, Lie algebras and equations of Korteweg-de Vries type, J. Sov. Math. 30 (1984) 1975 [INSPIRE].

[64] P. Saad, S.H. Shenker and D. Stanford, JT gravity as a matrix integral, arXiv:1903.11115 [INSPIRE]. 
[65] D.M. Hofman and B. Rollier, Warped Conformal Field Theory as Lower Spin Gravity, Nucl. Phys. B 897 (2015) 1 [arXiv:1411.0672] [INSPIRE].

[66] O. Coussaert, M. Henneaux and P. van Driel, The Asymptotic dynamics of three-dimensional Einstein gravity with a negative cosmological constant, Class. Quant. Grav. 12 (1995) 2961 [gr-qc/9506019] [INSPIRE].

[67] D. Hansen, J. Hartong and N.A. Obers, Action Principle for Newtonian Gravity, Phys. Rev. Lett. 122 (2019) 061106 [arXiv:1807.04765] [INSPIRE].

[68] N. Ozdemir, M. Ozkan, O. Tunca and U. Zorba, Three-Dimensional Extended Newtonian (Super)Gravity, JHEP 05 (2019) 130 [arXiv: 1903.09377] [INSPIRE].

[69] J. Gomis, A. Kleinschmidt, J. Palmkvist and P. Salgado-ReboLledó, Newton-Hooke/Carrollian expansions of (A)dS and Chern-Simons gravity, JHEP 02 (2020) 009 [arXiv: 1912.07564] [INSPIRE]. 